\title{
Disturbance-free BIST for Loop Characterization of \\ DC-DC Buck Converters \\ by
}

Priyanka Bakliwal

\section{A Thesis Presented in Partial Fulfilment of the Requirements for the Degree Master of Science}

Approved April 2015 by the Graduate Supervisory Committee:

Sule Ozev, Chair Bertan Bakkaloglu Jennifer Kitchen 


\begin{abstract}
Modern Complex electronic system include multiple power domains and drastically varying power consumption patterns, requiring the use of multiple power conversion and regulation units. High frequency switching converters have been gaining prominence in the DC-DC converter market due to their high efficiency. Unfortunately, they are all subject to higher process variations jeopardizing stable operation of the power supply.

This research mainly focus on the technique to track changes in the dynamic loop characteristics of the DC-DC converters without disturbing the normal mode of operation using a white noise based excitation and correlation. White noise excitation is generated via pseudo random disturbance at reference and PWM input of the converter with the test signal being spread over a wide bandwidth, below the converter noise and ripple floor. Test signal analysis is achieved by correlating the pseudo-random input sequence with the output response and thereby accumulating the desired behavior over time and pulling it above the noise floor of the measurement set-up. An off-the shelf power converter, LM27402 is used as the DUT for the experimental verification. Experimental results show that the proposed technique can estimate converter's natural frequency and Q-factor within $\pm 2.5 \%$ and $\pm 0.7 \%$ error margin respectively, over changes in load inductance and capacitance.
\end{abstract}




\section{DEDICATION}

Dedicated to my entire family and my dear friends! 


\section{ACKNOWLEDGMENTS}

Firstly, my heartfelt and sincere thanks to my humble and charming advisor Dr. Sule Ozev for providing me an opportunity to work on this valuable research project. Her guidance and support during my Master's and this project, both technically and emotionally has been amazing. I am deeply grateful to her.

I would like to thank Dr. Bertan Bakkaloglu for the immense support and encouragement during my Master's. I would also like to thank him and Dr. Jennifer Kitchen for kindly agreeing to be my thesis committee members and advising me through very insightful discussion throughout my Master's coursework.

I also like to thank all my research group members for their support.

My sincere thanks to James Laux for helping me out with all the computer and CAD support during the course of the project. He has responded very keenly to all the issues I faced in this regard.

Finally, I would like to thank my dear Uncle, my entire family and my dear cousins for their endless support, care, encouragement and love during my entire career. 
Page

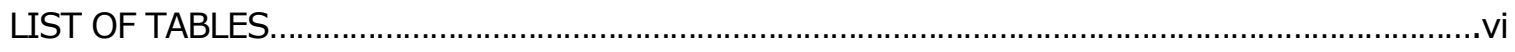

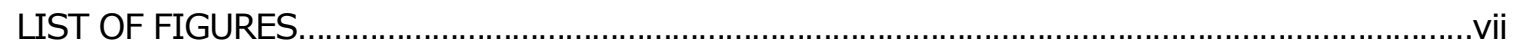

CHAPTER

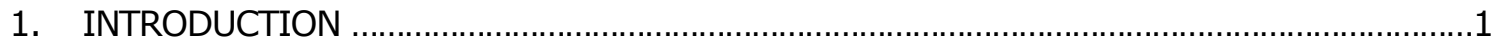

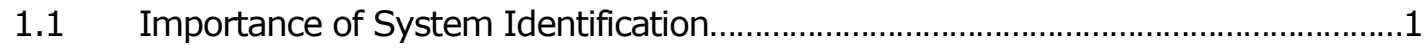

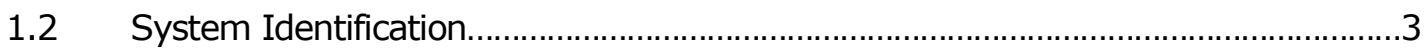

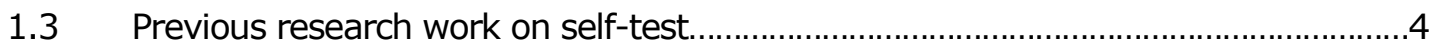

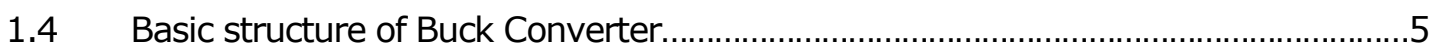

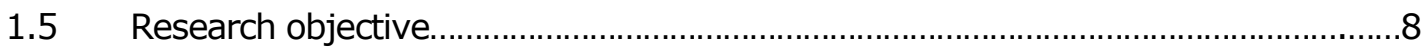

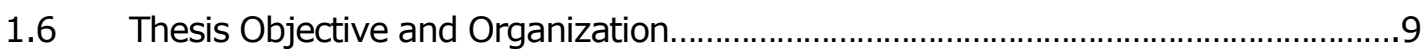

2. CORRELATION BASED DYNAMIC LOOP CHARACTERIZATION .........................................10

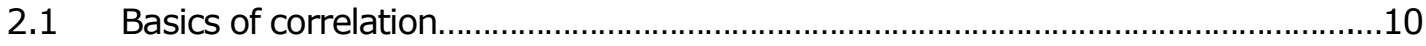

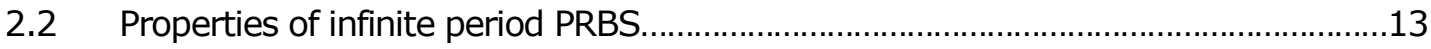

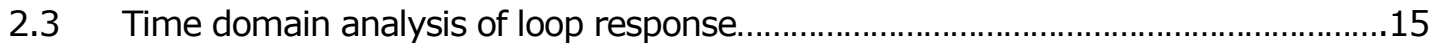

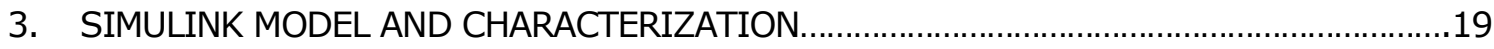

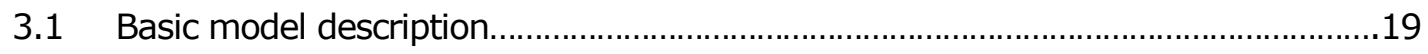

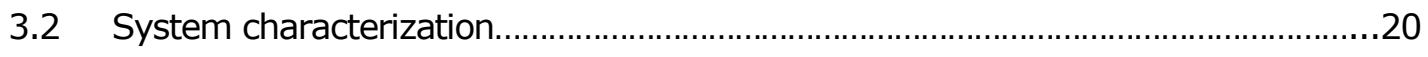

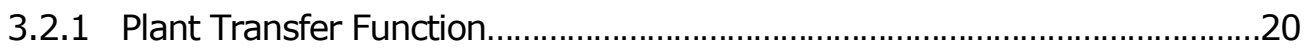

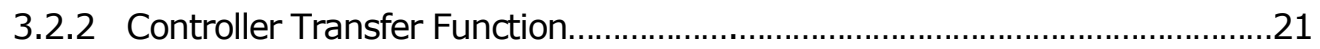

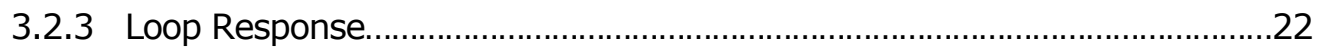

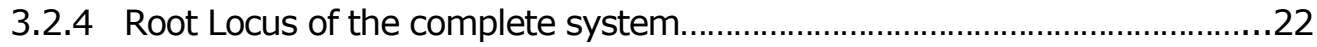


3.2.5 White noise based PRBS auto-correlation ................................................23

3.2.6 Cross-Correlation of PRBS with Vout by injection of PRBS at Vc node.........23

3.2.7 FFT plot of Cross-Correlated result and the Bode plot.................................24

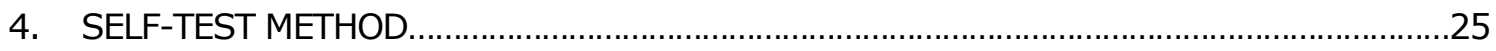

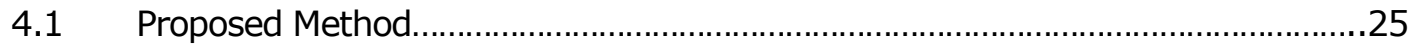

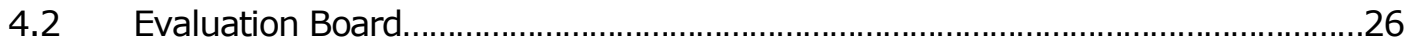

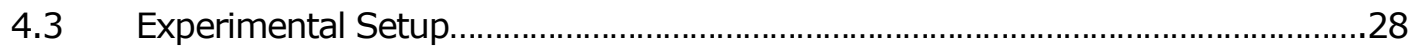

4.4 Transfer function from Reference Input to Output.............................................30

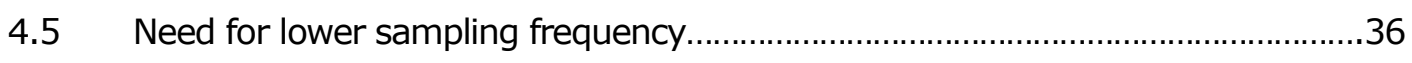

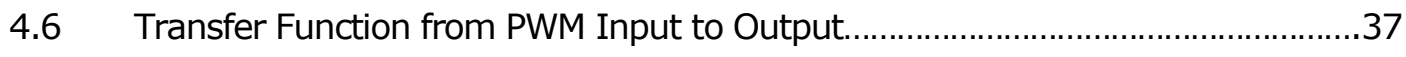

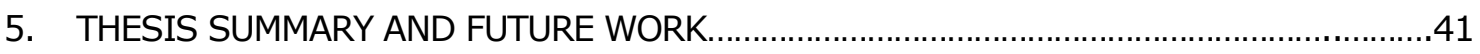

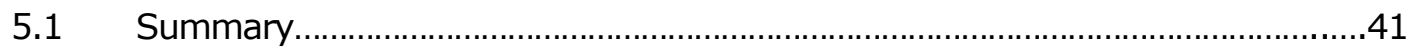

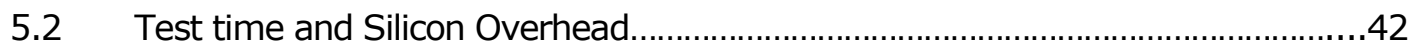

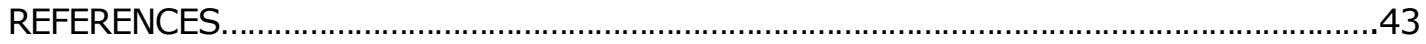




\section{LIST OF TABLES}

Table

Page

4.3.1 Load Inductances and Capacitances for experiment 29

4.4.1 $\omega_{\circ}$ and $Q$, measured for different output filter Inductances...........................................33

4.4.2 $\omega_{\circ}$ and $Q$, measured for different output filter Capacitances..........................................33

4.6.1 $\omega_{c}$ measured for different output filter Inductances...................................................39

4.6.2 $\omega_{c}$ measured for different output filter Capacitances...................................................40 


\section{LIST OF FIGURES}

Figure

Page

1.1.1 Impact of output filter variation on the open loop AC response of a typical DC-DC

Converter

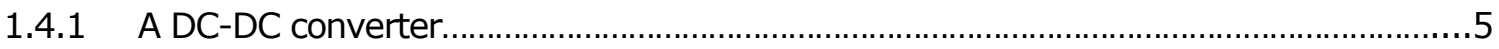

1.4.2 The buck converter with a current controller and a voltage controller..........................6

1.4.3 The waveform of the control signal and the inductor current.....................................

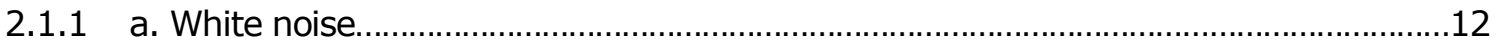

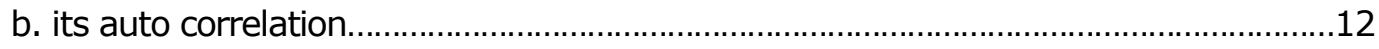

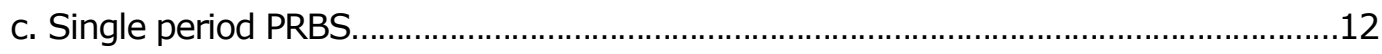

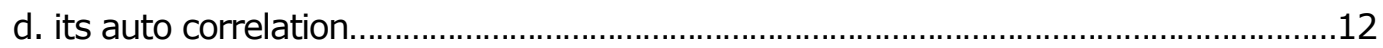

2.1.2 9-bit PRBS generated by a 9-bit shift register........................................................13

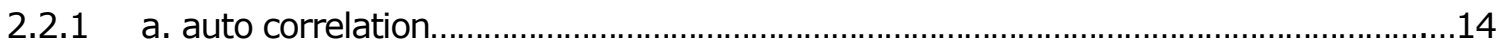

b. frequency spectrum of an infinite period PRBS.....................................................14

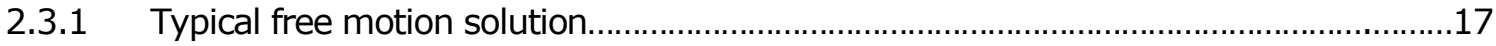

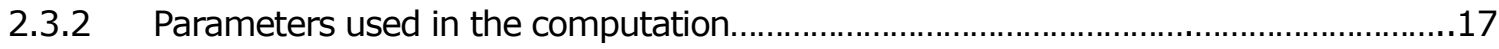

3.1.1 Simulink Model for System Identification of Buck Converter using PRBS...................19

3.2.1 Bode plot of the Plant

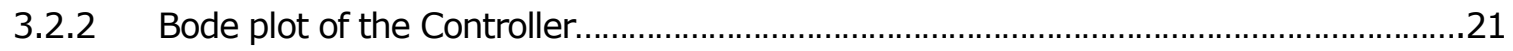

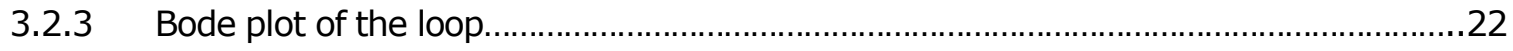

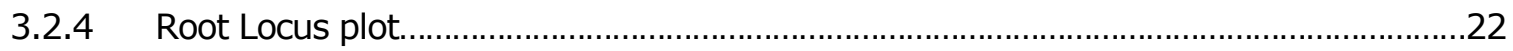

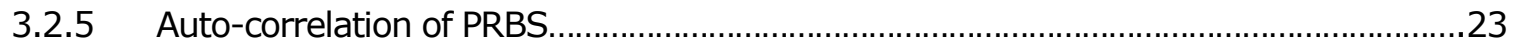

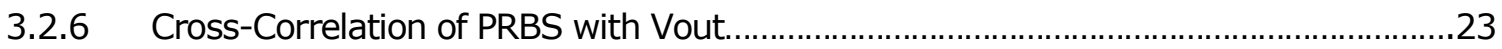

3.2.7 Bode plot using transfer function and FFT of the time domain impulse using PRBS..24

4.1.1 Proposed dynamic loop characterization methodology..............................................26 
4.2.1 Evaluation board LM27402 and the experimental set-up........................................27

4.2.2 Simplified block diagram of DC-DC converter on LM27402 Evaluation board with external System Identification blocks.....................................................................2

4.3.1 LCR low-pass filter showing discrete-time to continuous-time Interface....................29

4.3.2 Block diagram showing the continuous-time plant with the discrete time input and output.

4.4.1 Frequency response for different Inductance value using PRBS.................................31

4.4.2 Comparison between PRBS and Sine-Wave method................................................32

4.4.3 Swept Sine-Wave response of the system from Vref to Vout.....................................32

4.4.4 Spectrum of Output Voltage (Vout) with and without PRBS injection at converter reference Input (SS/TRACK) for correlation based System Identification..................34

4.4.5 Frequency response for different capacitance value using PRBS................................35

4.4.6 Response using Swept Sine-Wave with various capacitors.........................................35

4.4.7 Overlapping plot showing the PRBS and Swept Sine-Wave response........................36

4.6.1 PWM to Vout transfer function response using PRBS with varying inductance Value 38

4.6.2 PWM to Vout transfer function response using PRBS with varying capacitance value 38

4.6.3 Comparison between PRBS and Sine-Wave method. 39 


\section{CHAPTER 1 \\ INTRODUCTION}

\subsection{Importance of System Identification}

Switching mode DC-DC converters have been widely used as an integral part of Power Management Integrated Circuits (PMICs) and Power Management units (PMUs) in computer, communications and consumer electronics. Quite often, electronic systems and SOCs contain many Dc-Dc converters to supply multiple voltage domains, current/voltage requirements of which may change dynamically. As an example, the Haswell processor has thirteen switching DC-DC converters [1]. In order to satisfy fast response requirements with small form factor, the trend is to employ higher frequency switching. The switching frequency of Haswell DC-DC converters is $140 \mathrm{MHz}$ [1]. Dynamic performance and stability of DC-DC converters greatly depend on the overall loop characteristics. Loop dynamics in turn ae determined primarily by off-chip output filter inductance ( $L)$, inductor Dc resistance (DCR), load capacitance (C), capacitor equivalent series resistance (ESR), and the resistance of the power train transistors (Rds). In [1], converters with small output L-C filter are demonstrated using package trace inductors. Design of such high switching rate converters become a bigger challenge as the smaller form-factor output filter (LCR filter) suffers from higher manufacturing variations. For an example, average DCR of Vishay IHLP-5050FD $4.7 \mu \mathrm{H}$ inductor is about $9.32 \mathrm{~m} \Omega$ with $1.3 \%$ manufacturing 3-sigma variation and the average DCR of Vitec 59P9022 $100 \mathrm{nH}$ inductor is $0.3 \mathrm{~m} \Omega$ with $14.8 \%$ manufacturing 3 -sigma variation. 
In addition to the manufacturing variations, temperature and aging also cause drift in the converter loop. In [2], it has been shown that for a typical DC-DC converter, open loop frequency response phase margin drops by $13^{\circ}$ caused by $\pm 25 \%$ variation in the output filter and load current (see Figure 1.1.1).

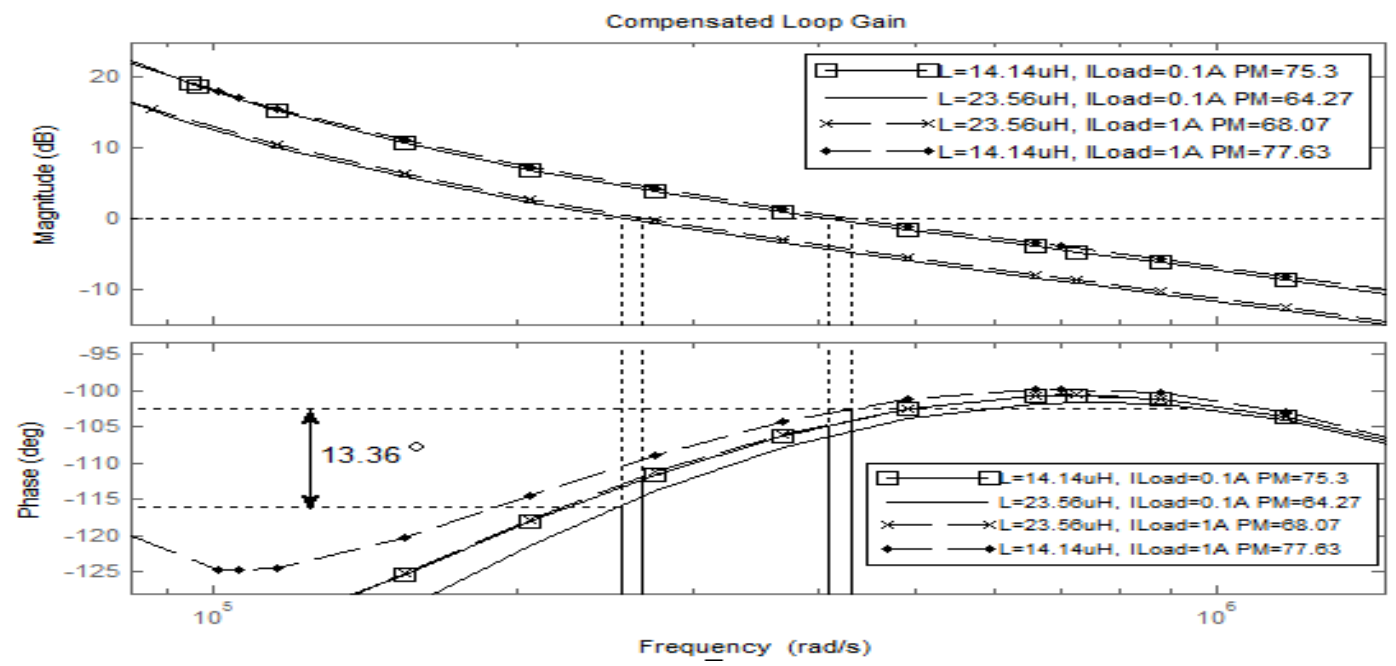

Figure 1.1.1: Impact of output filter variation on the open loop AC response of a typical DC-DC converter [2].

In order to ensure efficient and stable operation of the Dc-DC converter, dynamic loop characteristics need to be determined and the controller needs to be tuned with respect to these characteristics. Since the loop characteristics may shift, albeit incrementally and gradually, over time and environmental conditions, this monitoring and tuning cannot be done only after manufacturing, but it needs to be repeated in the field, while the device is actively working. Any in-filed measurement technique for the loop characteristics requires several aspects: (a) self-test needs to be transparent with respect to the normal operation of the converter, (b) the measurement needs to be conducted within the closed-loop and at operation point, 
(c) output response needs to be large enough to be immune to noise, and

(d) the measurement needs to present with little to no computational overhead.

These requirements contradictory as measurement requires an observable response to a specific test input, and an observable by the very definition disturbs the operation of the converter. Analysis of the observed response also typically requires extensive computational resources that are not available on the DC-DC converters.

\subsection{System Identification}

In general, characterization of the transfer function of a given circuit is also known as the system identification problem. The methods for system identification can be categorized as parametric and non-parametric [3],-[4]. Parametric identification methods start with a known structural model of the system and estimate the model parameters based on the observed response. Non-parametric methods do not assume any particular model structure, but incrementally determines the time or frequency domain impulse response by exciting the system with known inputs and directly observing its response. Existing non-parametric methods are transient analysis [5], frequency response analysis [6], and correlation analysis [7], [8].

Two well-known transient methods are the step-input injection and impulse injection methods. Injection of a sine-wave input and measuring the gain of the system for different input frequencies is a standard transfer function analysis method. Unfortunately, such system identification methods do not satisfy the above mentioned requirements since they disturb the operation of the converter. 


\subsection{Previous research work on self-test}

A number of methods have been proposed to self-test DC-Dc converters at production time. An open-loop control-to-output system identification technique using pseudo random input stimulus is proposed in [10]. Due to its open loop mode requirement, this technique is not suitable for on-lie characterization of DC-DC converters.

In [9], the authors have proposed a safe test method to correlate the converter parameters under high and low current scenarios for PMUs. Similar statistical modeling approaches, as in [9], can also be used for the loop characterization. However, they too need an observable response at the output.

In [11], a Gaussian windowed closed-loop based transfer function measurement method is proposed using pseudo random input signals. The goal in [10], [11] is different than in-field self-test, it is to improve the higher frequency response of the system. As such, higher frequency observation is necessary, which complicates the self-test problem. A multi-period maximum length pseudo random binary sequence

(MLBS) is used as the excitation signal in [12]. The transfer functions are identified from the measurement data with circular cross-correlation method. However, this technique uses digital low pass filtered MLBS, making it a multi-level analog excitation, therefore this technique requires a full D/A converter to generate the MLBS and a transformer to couple the signal to the regulator output. 


\subsection{Basic Structure of Buck Converter}

Figure 1.4.1 shows a dc-dc converter as a black box. It converts a dc input voltage, $v g(t)$, to a dc output voltage, $v o(t)$, with a magnitude other than the input voltage (Erickson and Maksimovic, 2000, Section 1.1). The converter often includes one (or several) transistor(s) in order to control the output voltage, using the control signal $\delta(t)$.

It is desirable that the conversion be made with low losses in the converter. Therefore, the transistor is not operated in its linear interval. Instead, it is operated as a switch and the control signal is binary. While the transistor is on, the voltage across it is low which means that the power loss in the transistor is low. While the transistor is off, the current through it is low and the power loss is also low. To obtain low losses, resistors are avoided in the converters. Capacitors and inductors are used instead since ideally they have no losses.

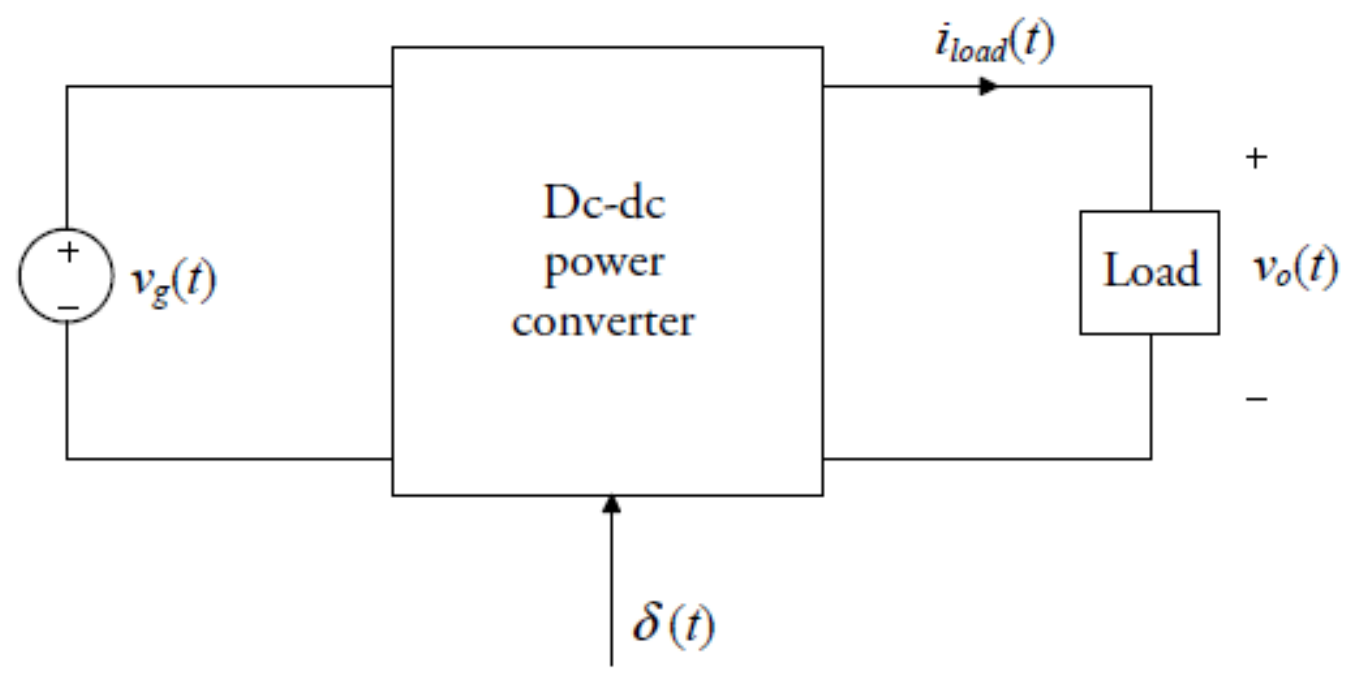

Figure 1.4.1: A DC-DC Converter 
The electrical components can be combined and connected to each other in different ways, called topologies, each one having different properties. The buck, boost, and buck-boost converters are three basic converter topologies. The buck converter has an output voltage that is lower than the input voltage. The boost converter has an output voltage that is higher than the input voltage (in steady state). The buck-boost converter is able to have an output voltage magnitude that is higher or lower than the input voltage magnitude.

Figure 1.4.2 shows the buck converter with two controllers. Here it is assumed that all components are ideal. The load consists of a resistor with resistance $R$. The converter has a low-pass output filter consisting of an inductor with inductance $L$ and a capacitor with capacitance $C$. While the transistor is on, the inductor current, $i_{L}(t)$, increases since the input voltage is higher than the output voltage in the buck converter. As the transistor is turned off, the diode must start to conduct since the inductor current cannot stop flow instantaneously. The voltage across the diode is

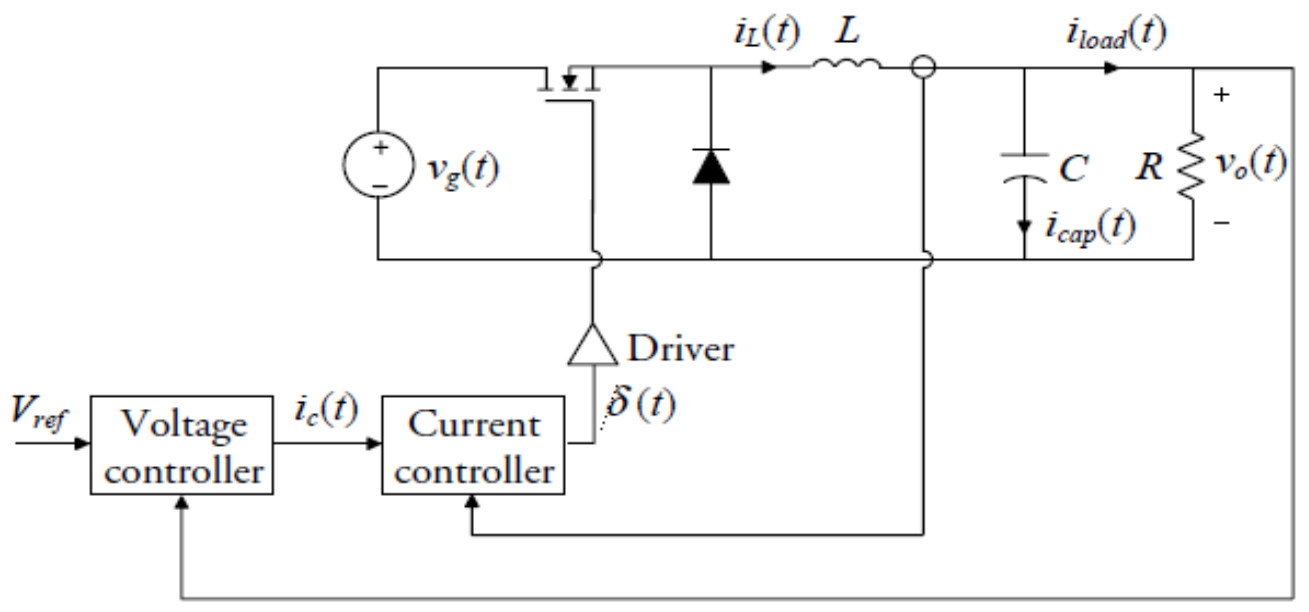

Figure 1.4.2: The buck converter with a current controller and a voltage controller

zero when it is conducting and the inductor current will decrease. 
Figure 1.4.3 shows the waveforms of the control signal and the inductor current. The converter is usually designed so that the magnitude of the ripple in the output voltage becomes small. If the ripple is insignificant, the inductor current increases and decreases linearly as shown in Figure 1.4.3

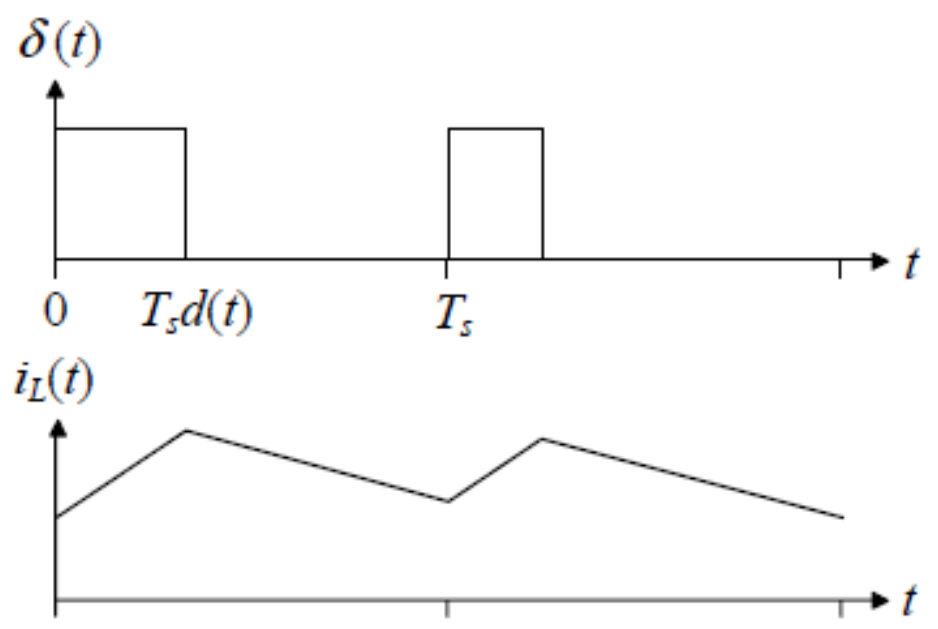

Figure 1.4.3: The waveforms of the control signal and the inductor current

The voltage across the diode is equal to the input voltage or equal to zero. The output filter of the converter filters this voltage waveform and the magnitude of the ripple in the output voltage depends on the filter design. If the inductor current becomes zero before the transistor is turned on, it will remain at zero until the transistor is turned on since the diode can only conduct in one direction. If the converter is operated so that the inductor current is zero during some part of the switching period, it is said to be operated in discontinuous conduction mode.

Otherwise, it is operated in continuous conduction mode.

The switching period, Ts, of the converter is determined by the control signal $\delta(t)$, as shown in Figure 1.4.3. In this figure, the switching period is held constant. The average output voltage is controlled by changing the width of the pulses. 
In Figure 1.4.3, the falling edge is controlled i.e. when the transistor should turn off. The duty cycle, $d(t)$, is a real value in the interval 0 to 1 and it is equal to the ratio of the width of a pulse to the switching period. The control signal $\delta(t)$ can be obtained from $d(t)$ by using a pulse width modulator. The duty cycle can be seen as a discrete-time signal.

\subsection{Research Objective}

This research aims at developing innovative technique to determine the closed-loop transfer function of the DC-DC converters without significantly affecting its output noise beyond the existing ripple. Concept of spectrum spreading techniques (such as CDMA) is used in the communications domain to hide the test input/output signals with the existing noise floor of the DC-DC converter. A small perturbation pseudo random signal at an accessible input node of the converter (i.e. the reference and pulse width modulator input). While the output response is not directly measurable. To see the system response, correlation of the input and output signals is done to accumulate over time, thereby pulling it to above the noise floor. Aim is to obtain the impulse response of the closed-loop operation. This impulse response (or the frequency domain equivalent, transfer function) can be analyzed to determine important stability characteristics, such as settling time, overshoot, and undershoot. In contrast to a previous similar technique [12], the proposed approach uses only a binary pseudo random bit sequence, doesn't require analog coupling, extracts stability parameters directly from measurements without resorting to computationally intensive procedures, and the work within the closed-loop system, making it more suitable for fully integrated DC-DC converters. 


\subsection{Thesis Outline and Organization}

This research thesis report is organized into 5 chapters. Chapter 1 establishes the need for system identification and introduces a generic Buck Converter structure. It also gives the details on the research objective and background.

Chapter 2 introduces the White noise based characterization. It discusses the PRBS based injection and its similarity with White noise.

Chapter 3 gives the detail of Simulink model of Buck Converter and its characterization using 10-bit PRBS. This section also has Simulink results with variation in component values which can be detected by PRBS.

Chapter 4 capture of the chip characterization results and compare it with Simulink model. It section also points out practical limitation of sampling on board compared to simulation.

Chapter 5 discuss the future work and improvement. 
CHAPTER 2

\section{CORRELATION BASED DYNAMIC LOOP CHARACTERIZATION}

\subsection{Basics of correlation}

In steady state operation, for small-signal disturbances, a switching power converter can be approximated as a linear time-invariant sampled system [13]. A linear time-invariant sampled system can be described as

$$
y[n]=\sum_{k=1}^{\propto} h[k] x[n-k]+v[n]
$$

where $y[n]$ is the sampled output signal, $x[n]$ is the sampled input signal, $h[n]$ is the discrete-time system impulse response and $v[n]$ represents unwanted disturbances, such as switching noise, quantization noise, etc. The cross-correlation of the input signal $x[n]$ and the output signal $y[n]$ is as follows:

$$
\begin{aligned}
R_{x y}[m] & =\sum_{n=1}^{\alpha} x[n] y[n+m] \\
& =\sum_{n=1}^{\infty} h[n] R_{x x}[m-n]+R_{x v}[m]
\end{aligned}
$$

where $R_{x y}[\mathrm{~m}]$ is the cross-correlation of input and output signals, $R_{x x}[\mathrm{~m}]$ is the autocorrelation of input signal and $R_{x v}[\mathrm{~m}]$ is the cross-correlation of input signal with disturbances [3]. Now, if $x[n]$ is white noise, then correlation functions $R_{x x}$ and $R_{x v}$ have the following properties:

$$
R_{x x}[m]=\delta[m]
$$




$$
R_{x v}[m]=0
$$

where $\delta[\mathrm{m}]$ is an ideal delta function. Auto-correlation of white-noise input is ideal delta function and cross-correlation of white-noise input with unwanted disturbances $v[n]$ is ideally zero. This simplifies Eq. (2) and the cross-correlation becomes the discrete-time system impulse response [3]. The discrete Fourier transform (DFT) of impulse response gives system frequency response.

$$
\begin{aligned}
& R_{x y}[m]=h[m] \\
& R_{x y}[m] \stackrel{D F T}{\longrightarrow} H[j \omega]
\end{aligned}
$$

The properties presented in Eq. (3)-(4) need the injection signal to be white noise. In addition, it is desirable that the signal generation adds low overhead. In practical implementation, an approximate white noise is generated by pseudo random binary sequence (PRBS) generator consisting of shift registers and feedback taps [14]. The PRBS is periodic and deterministic, and the data length of the n-bit maximum length PRBS generator is given by $M=2^{n}-1$.

Auto-correlation of white-noise is an ideal delta, but for PRBS, auto-correlation function is a mix of a delta function at $m=0$ and low amplitude components at $m \neq 0$. Similarly, the cross-correlation of PRBS with system disturbances $v[n]$ is not zero. Hence, the cross-correlation $\left(R_{x y}\right)$ of input PRBS with output signal has undesired noise terms in addition to the system impulse response due to the non-ideality in $R_{x x}$ and $R_{x v}$ of Eq.(3). 
Figure 2.1.1 shows a comparison of white noise (a) and a 9-bit single period PRBS (c) in a digital system. Figure 2.1 (b) and (d) show the corresponding autocorrelation functions, respectively. We can see that the auto-correlation of a single period PRBS is very close to a delta function, but now with a no-ideal component

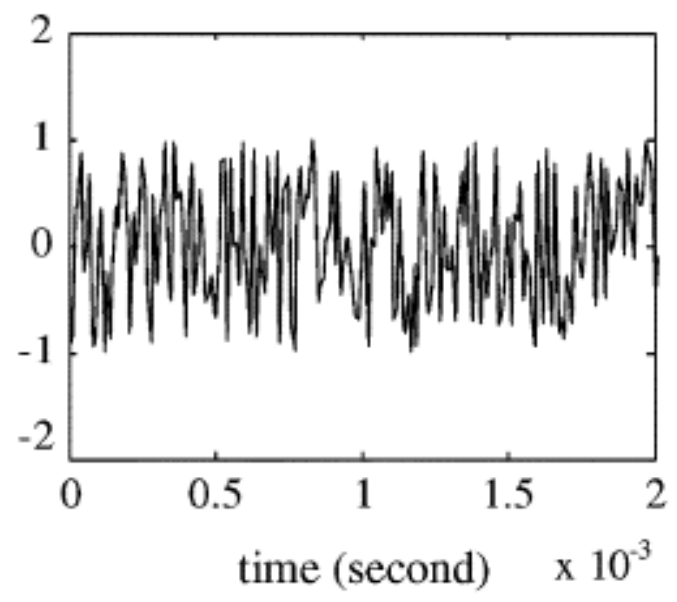

(a)

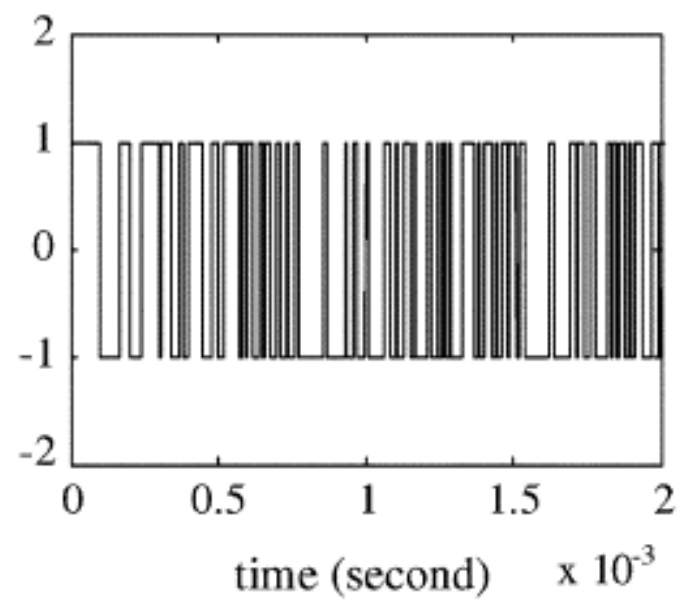

(c)

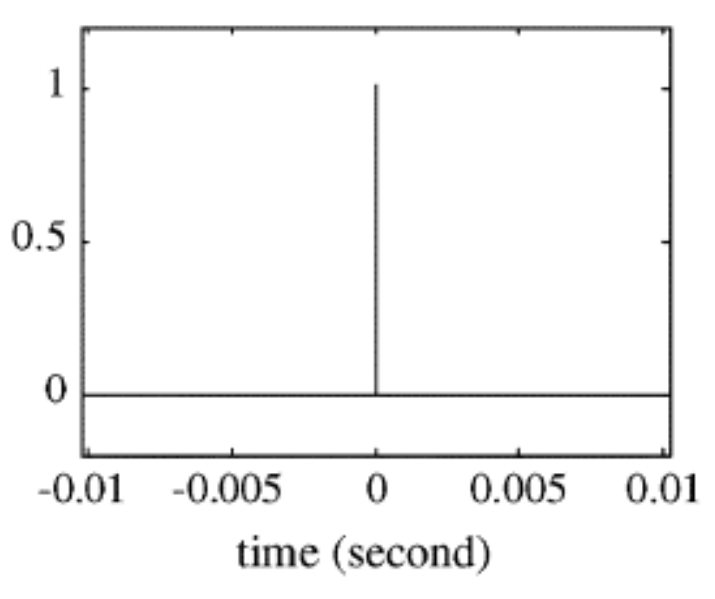

(b)

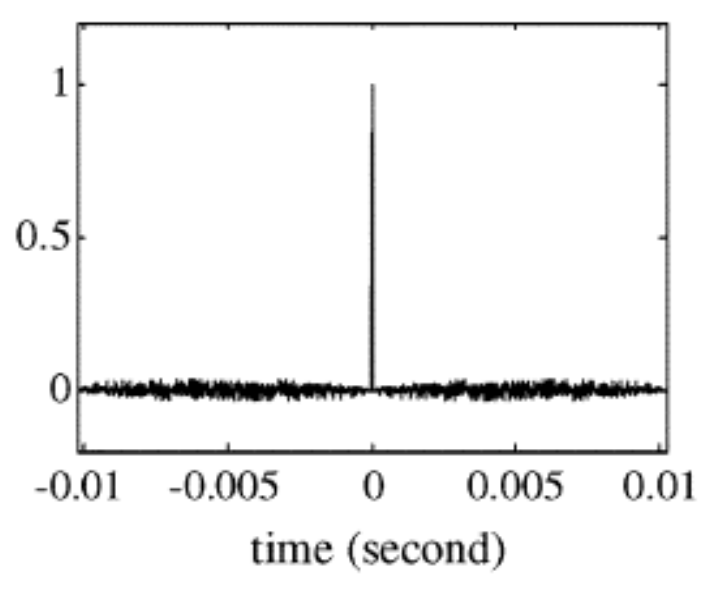

(d)

Figure 2.1.1 (a) White noise and (b) its auto correlation. (c) Single period PRBS and (d) its auto correlation. Sampling frequency is $100 \mathrm{kHz}$.

(or noise) around it. Recall from (2) that the cross-correlation between the input and 
output can be seen as time convolution between the auto-correlation of the input (ideally a delta function) and the system impulse response. The additional noise in the PRBS autocorrelation will create errors in our identification process.

The PRBS perturbation signal can be easily generated in a digital system using a shift register with feedback, as shown in Figure 2.1.2 for a 9-b PRBS. An n-bit feedback shift register can generate several different sequences, among which the

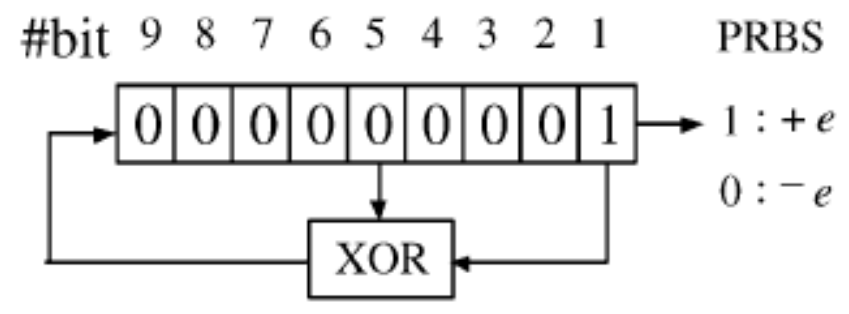

Figure 2.1.2: 9-b PRBS generated by a 9-b shift register

maximum length sequence has the best properties (optimal noise-like characteristics) for this application. The maximum length PRBS can be generated by performing an XOR operation between the $i$-th bit and a specific $j$-th bit. For a 9-b shift register, the XOR operation should be performed between the first and the fifth bits, as shown in Figure 2.1.2. The output generated by a one-bit right shift operation produces a maximum length sequence of 511 before repetition occurs.

\subsection{Properties of Infinite period PRBS}

A maximum length PRBS repeated $L$ times forms an $L$-period PRBS. If $L$ tends to infinity, it has the following properties and frequency spectrum:

$$
\left|\frac{1}{M} \sum_{k=1}^{M} u(k)\right|=\frac{e}{M}
$$




$$
\begin{aligned}
& R_{u u}(k)=\frac{1}{M} \sum_{j=1}^{M} u(j) u(j+k) \\
& =\left\{\begin{array}{lr}
e^{2,} & k=0, \pm M, \pm 2 M . . \\
\frac{-e^{2}}{M}, & \text { else }
\end{array}\right. \\
& \mu_{u}(f)=\frac{C e}{M} \sum_{k=1}^{M-1} \delta\left(f-\frac{k}{M} f_{o}\right), 0 \leq f \leq f_{o} .
\end{aligned}
$$

Equation (5) gives the mean value of an infinite period PRBS, which tends to zero for large M. Interestingly, (6) shows a key result: for an infinite period PRBS, the autocorrelation is given by periodic delta functions with magnitude $e^{2}$ at $k$ equal to zero and multiples of $\mathrm{M}$, and equal to $e^{2} / \mathrm{M}$ for all the other $k^{\prime} \mathrm{s}$, which is also shown in Figure 2.2.1(a) When $M$ is large, $e^{2} / M \rightarrow 0$, resulting in a periodic sequence of near ideal delta functions in the auto correlation.

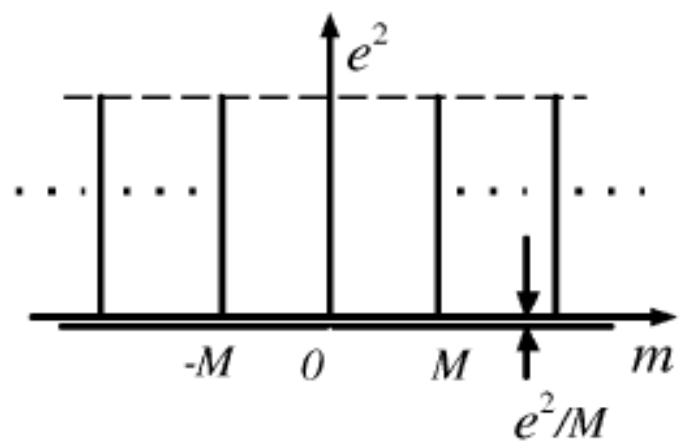

(a)

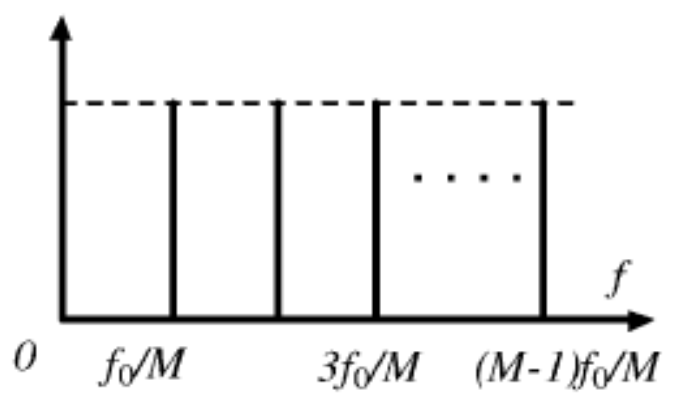

(b)

Figure 2.2.1: (a) Auto correlation and (b) frequency spectrum of an infinite period PRBS 
Figure 2.2.1(b) shows a plot of (7), where it is seen that the frequency content of an infinitely repeating PRBS contains delta functions of $k . f_{o} / M$ for $k=1, \ldots \ldots, M-1$, where $f_{o}$ is the frequency of PRBS. Thus the infinitely repeating PRBS can be seen as equivalent to injecting signals at M-1 discrete frequencies $k . f_{o} / M$, resulting in a clear limitation to the frequency components that can be identified in the power converter. In comparison, injection of white noise results in a flat line in the frequency domain, or is equivalent to signal injection at all frequencies for ideal system identification. Thus, for large $M$, an infinitely repeating PRBS injection would result in near ideal identification.

In practice, due to limitations in memory and computation capability, only finite length data can be used. However, we see significant improvement by decreasing the PRBS frequency or expanding them in time domain. Noise is also reduced by using circular correlation, where the effect of zero padded ends and heads of the linear correlation procedure is reduced by circulating the two data sequences and multiplying the corresponding bits. Averaging and windowing after cross- correlation also helps a lot in noise reduction.

\subsection{Time domain analysis of the loop response}

Apart from frequency response characterization, non-parametric analysis is also carried out using time domain impulse response using PRBS. To determine the stability of a system we need to calculate the vibrational parameters by looking at the time domain impulse response. 
The undamped natural frequency $\omega_{\mathrm{n}}$ and the damping ration $\zeta$ can be determined from a plot of the free motion of the system. If the damping is light, $\zeta<0.2$, the logarithmic decrement can be used to determine the coefficients. Systems with moderate damping are those in the range of $0.2<\zeta<1.2$.

The logarithmic decrement is used to determine the vibrational parameters and it works very well for systems in which the damping ratio lies in the range of $0.2-1.2$. These systems are moderately damped. For moderately damped systems, the differential equation for the free motion is

$$
\ddot{x}+2 \zeta \omega_{n} \dot{x}+\omega_{n}^{2} x=0
$$

Suppose we take $t=0$ to be the time when the solution to $(x)$ has the value $x=0$.

Then the solutions to $(x)$ are

$$
\begin{array}{ll}
x=\left(\frac{v_{o}}{\omega_{d}}\right) e^{-\omega_{n \zeta} t} \sin \left(\omega_{d} t\right) & 0<\zeta<1 \\
x=v_{o} t e^{-\omega_{n} t} & \zeta=1 \\
x=\left(\frac{v_{o}}{\omega_{d}}\right) e^{-\omega_{n \zeta} t} \sinh \left(\omega_{d} t\right) & 1<\zeta
\end{array}
$$

Where $\omega_{d}=\omega_{n} \sqrt{\mid 1-} \zeta^{2} \mid$ and $v_{o}=\dot{x}(0)$. Figure 2.3.1 shows typical plots of the solutions (x1).

A number of points and measurements will be used in the calculations. These are identified in figure 2.3.2 The case shown is of a system with damping ratio $\zeta=$ 0.75 . 


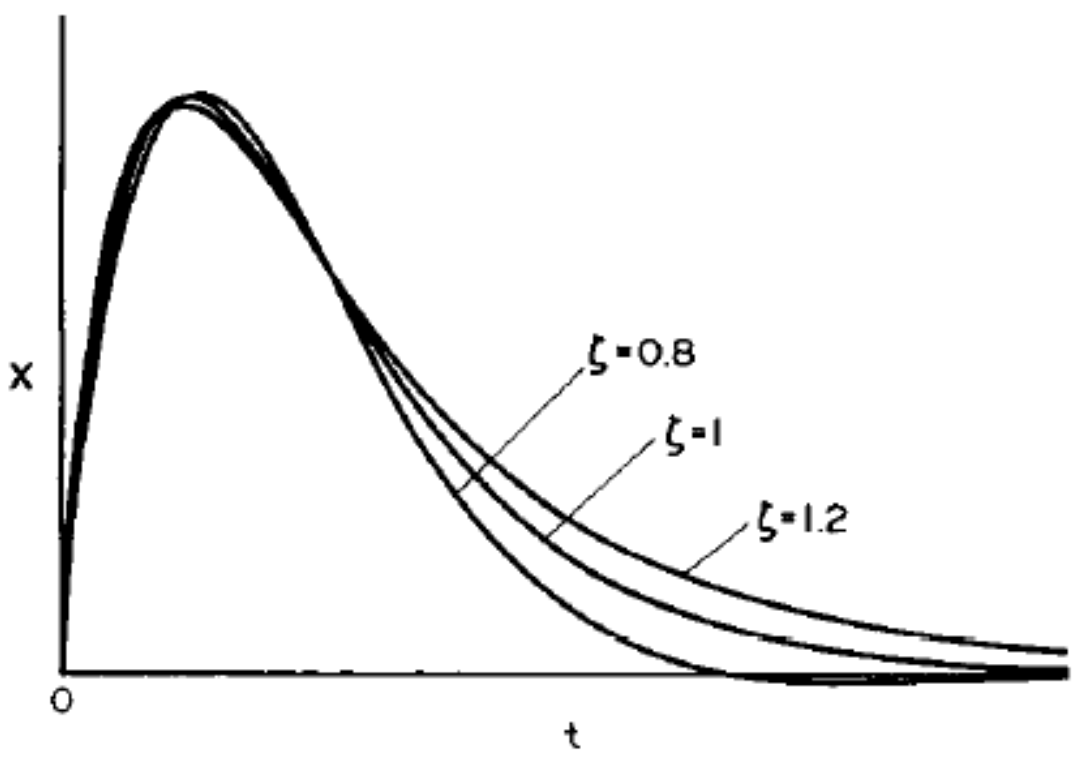

Figure 2.3.1. Typical free motion solution

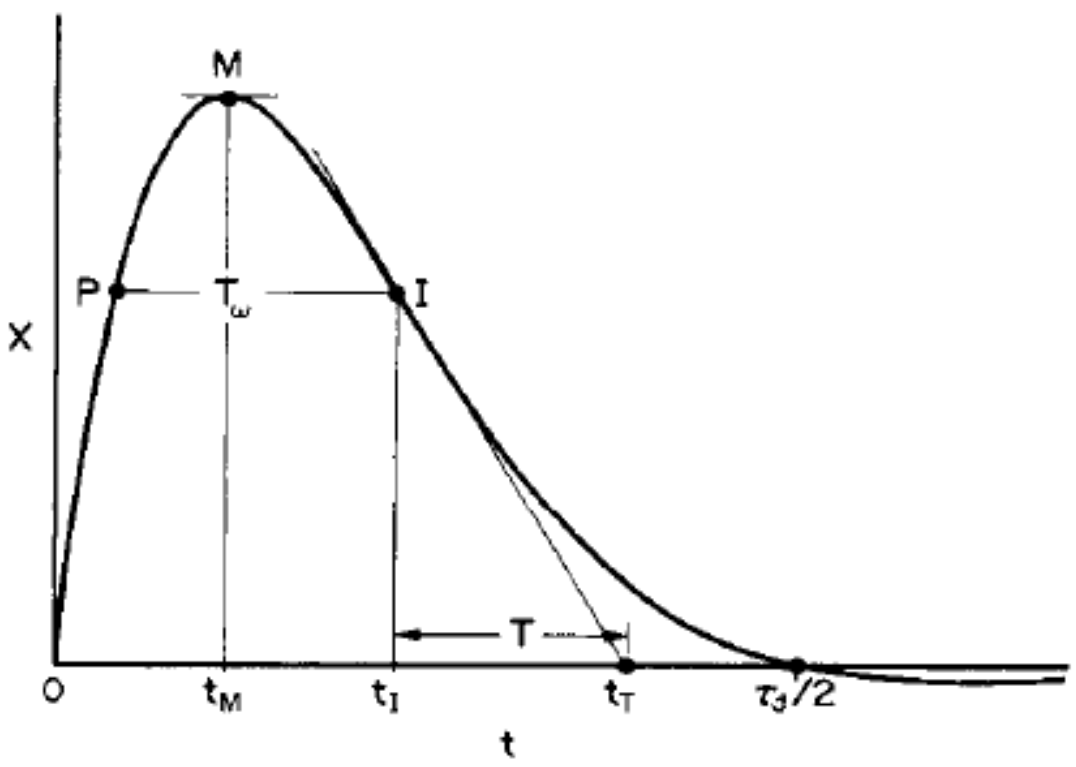

Figure 2.3.2 Parameters used in the computation

However, all except one of the parameters will be relevant for all cases of moderate damping. 
The following points and quantities will be used in the computation:

- M - a maximum point of the curve

- $\mathrm{XM}_{\mathrm{M}}$ - the displacement at the point $\mathrm{M}$

- $t_{m}$ - the time of the point $M$

- I - the inflection point after $M$

- $\mathrm{X}_{\mathrm{I}}$ - the displacement at the point I

- $t_{I}$ - the time of the point I

- $\mathrm{t}_{\mathrm{T}}$ - the time at which a tangent line through I crosses the time axis

- $\quad T$ - the time from $t_{I}$ until $t_{T}$

- P - a point preceding I at the same displacement as I

- $\mathrm{T}_{\omega}$ - the time from $\mathrm{P}$ to $\mathrm{I}$

- $\frac{\tau_{d}}{2}$ - the half period (for underdamped systems only). 


\section{CHAPTER 3}

\section{SIMULINK MODEL AND CHARACTERIZATION}

\subsection{Basic Model Description}

This chapter illustrates the behavior of a switched-mode converter with simulator examples. The Simulink model has Buck Converter which is been characterized in closed loop pattern using pseudo random binary sequence. Figure 3.1.1 shows the Simulink model of Buck Converter which has L-C filter at the output. Non idealities of inductor and capacitor is kept under consideration. Rdcr and Resr has been added to the model include it.

For characterization pseudo random sequence is used which is been generated using PN Sequence Generator block. Buck converter is characterized by injecting at Vc node. The Vc node covers the Plant in the forward path. This is the PWM injection point.

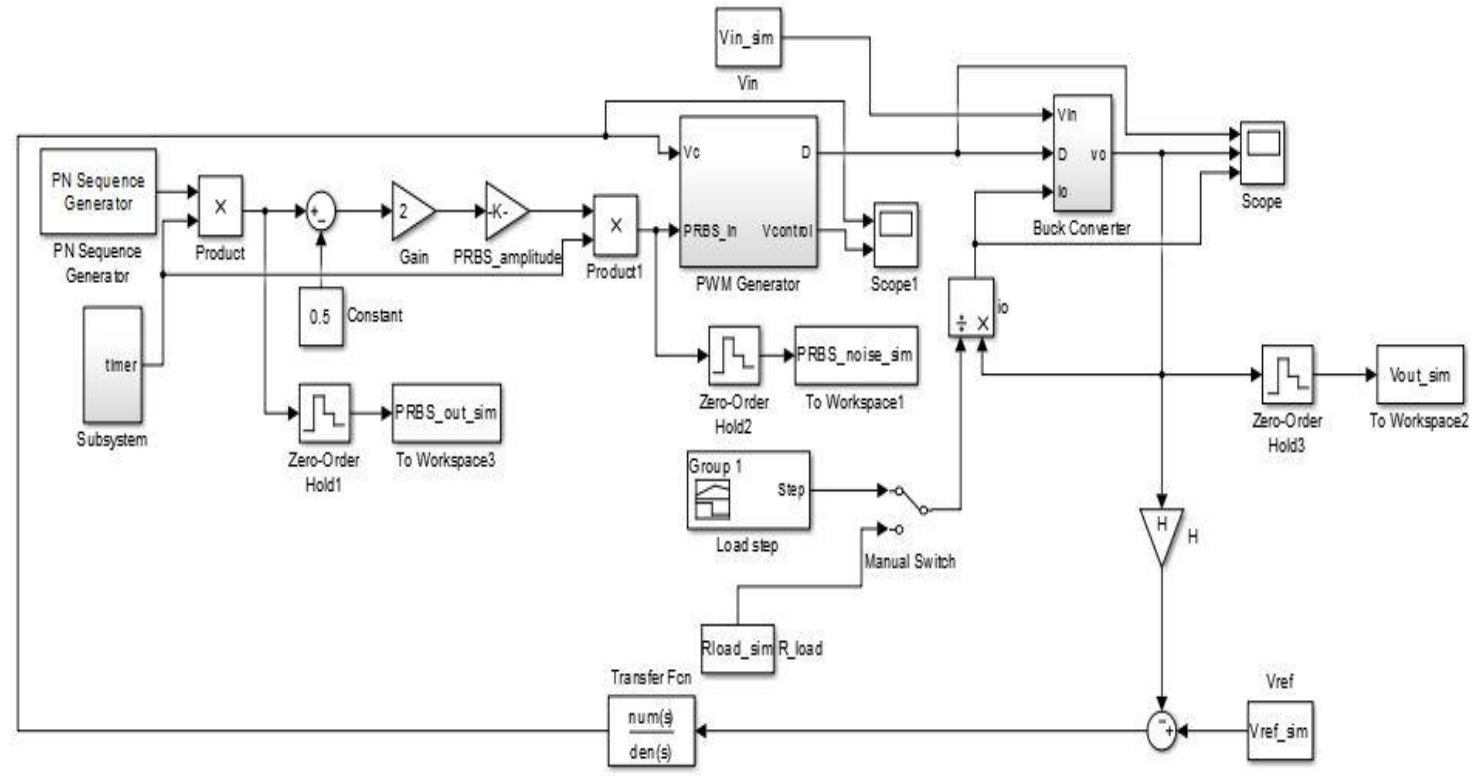

Figure 3.1.1 Simulink Model for System Identification of Buck Converter using PRBS 


\subsection{System Characterization}

The Buck Converter model has been designed with input voltage of $5 \mathrm{~V}$ which can go maximum value of $8.125 \mathrm{~V}$. The output voltage can be set to $3.3 \mathrm{~V}$ or $5 \mathrm{~V}$ according to the requirement. The model is designed to have an output current of 0.5A. A moderate switching frequency of $5 \mathrm{MHz}$ is used to design this converter. Plant transfer function in form of bode plot depicting the LC response.

\subsubsection{Plant Transfer Function}

LC filter of the plant with $\mathrm{L}$ as $3.91 \mu \mathrm{H}$ and $\mathrm{C}$ as $1 \mu \mathrm{F}$

Bode Diagram

$\mathrm{Gm}=\operatorname{lnf}, P \mathrm{Pm}=13.8 \mathrm{deg}($ at $1.38 \mathrm{e}+06 \mathrm{rad} / \mathrm{s})$

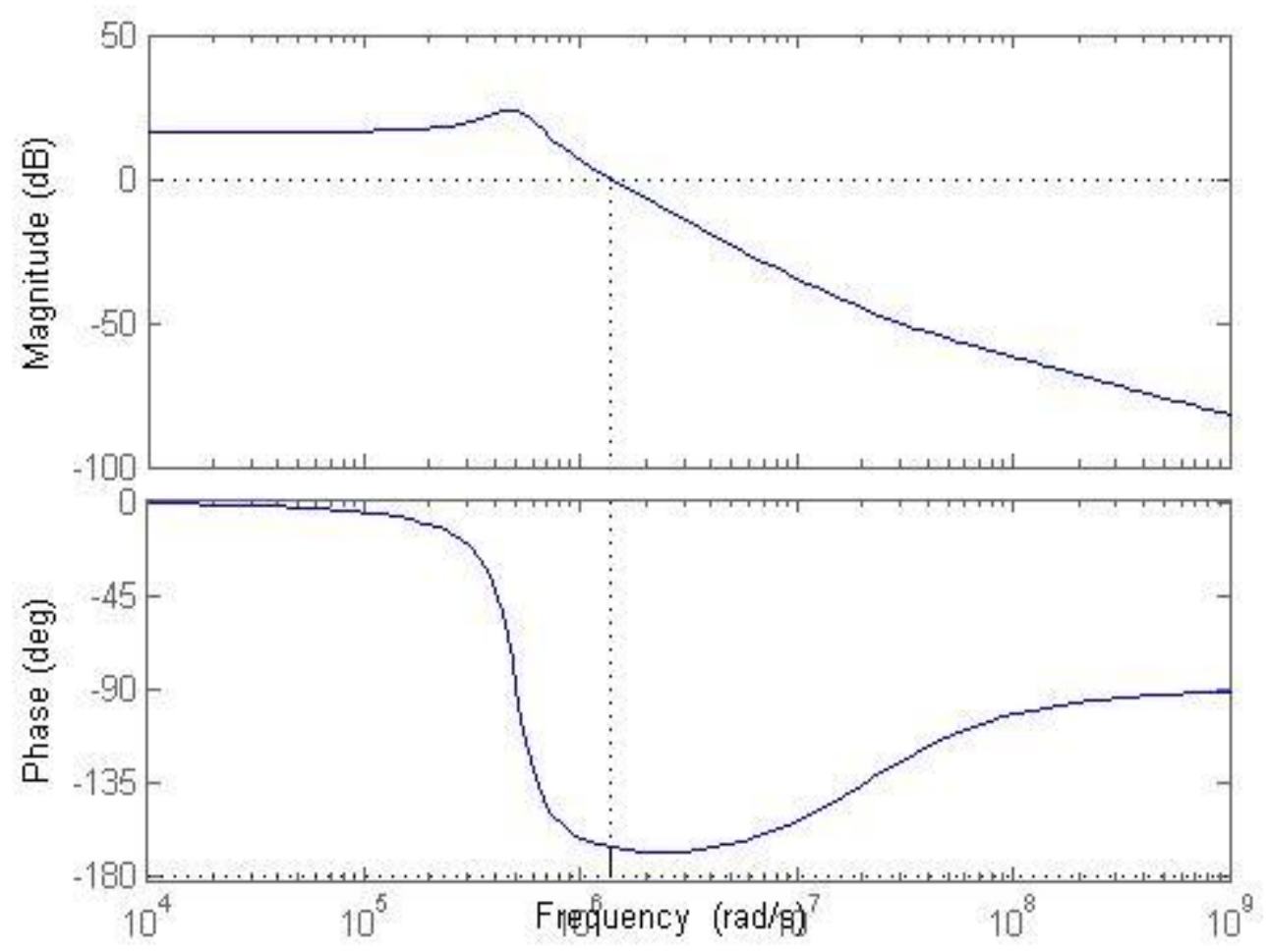

Figure 3.2.1 Bode plot of Plant 


\subsubsection{Controller Transfer Function}

Controller designed using pole-zero cancellation method to give enough phase margin to the system for stability.

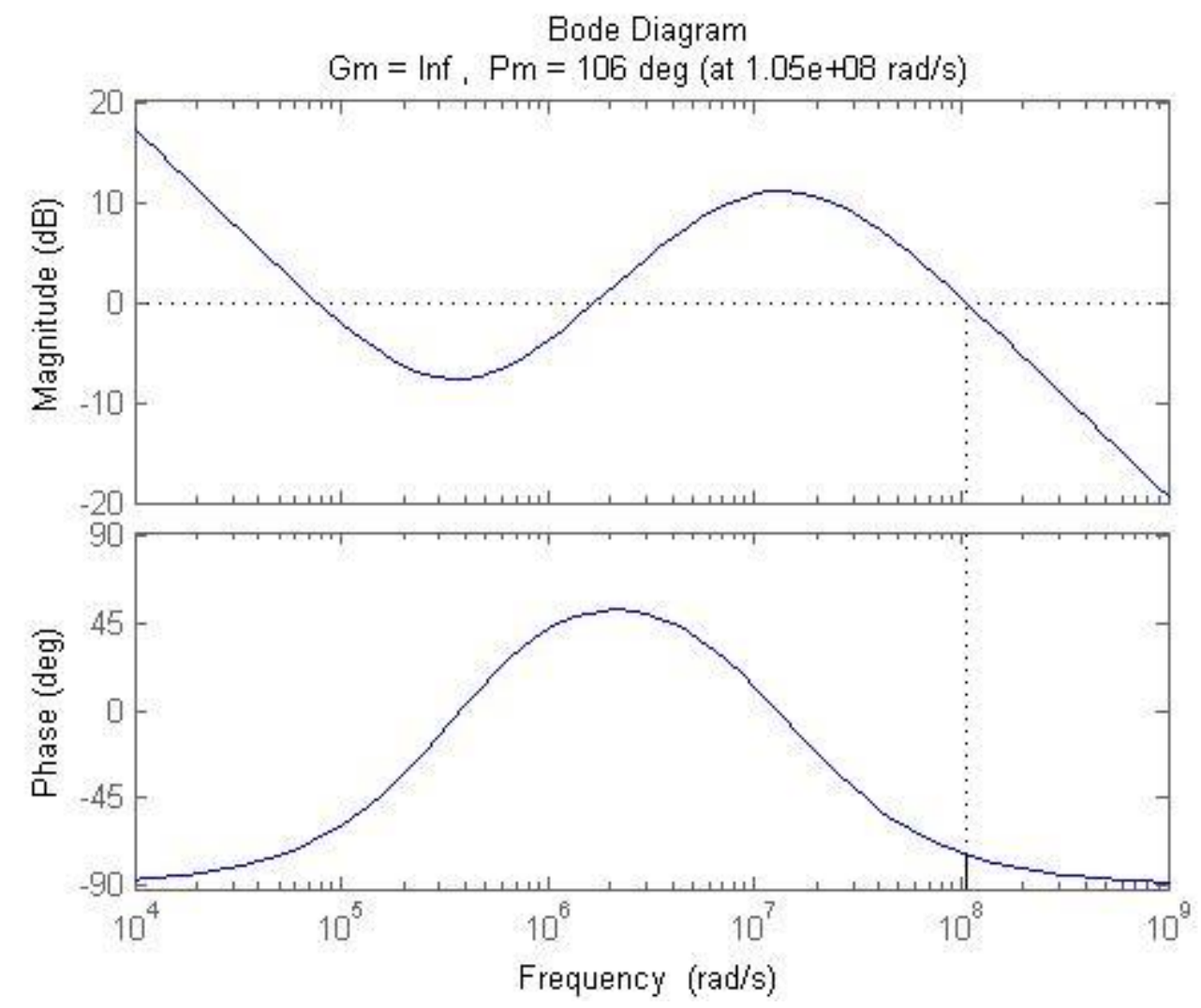

Figure 3.2.2 Bode plot of the Controller 


\subsubsection{Loop Response}

Loop response of the system with Phase margin of 90 degree.

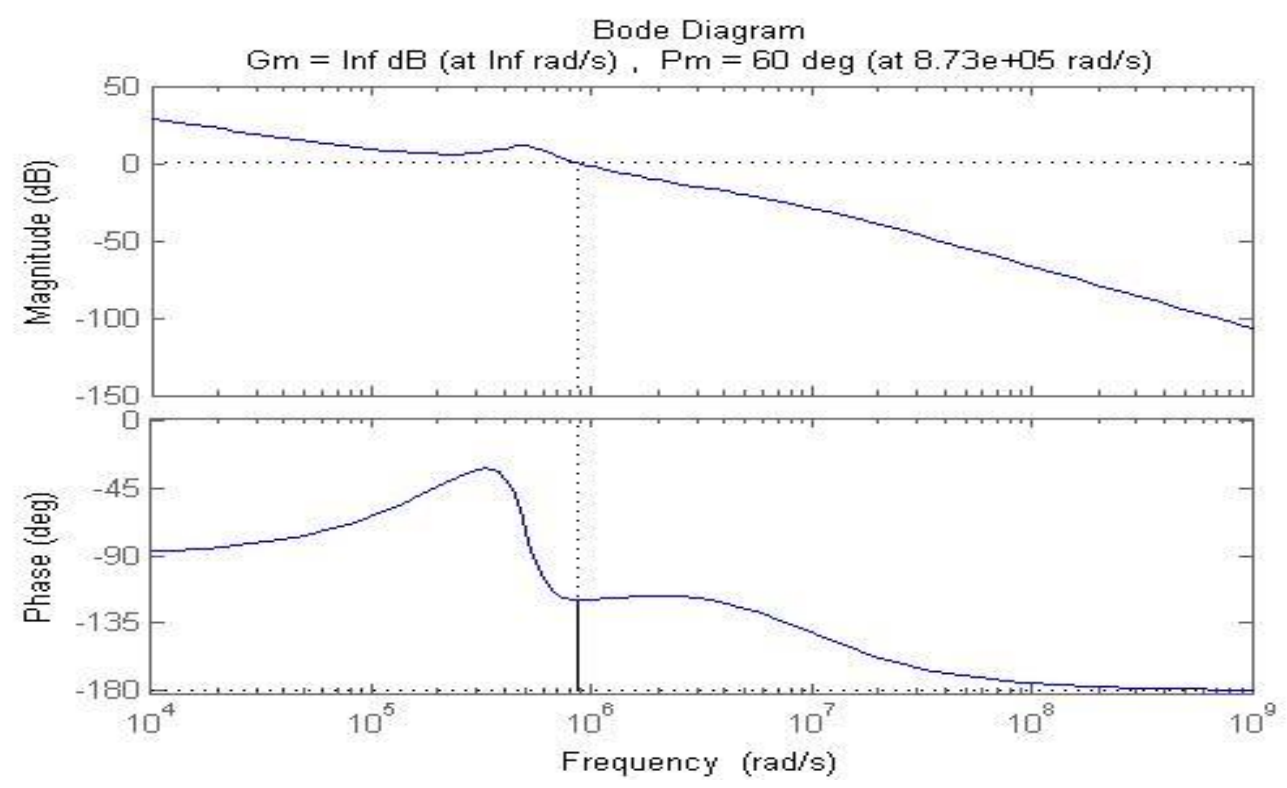

Figure 3.2.3 Bode Plot of the loop

\subsubsection{Root Locus of the complete system}

The plot showing the poles and zeros and they being in the left half plane shows the system is stable.

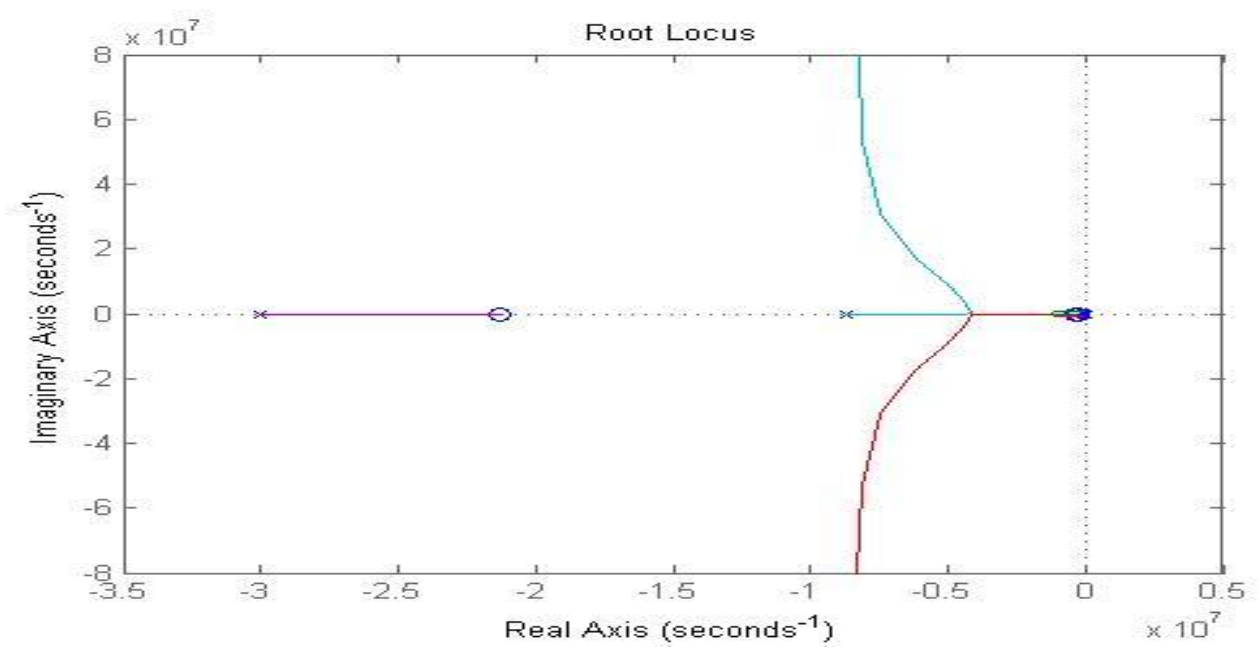

Figure 3.2.4 Root Locus plot 


\subsubsection{White noise based PRBS auto-correlation}

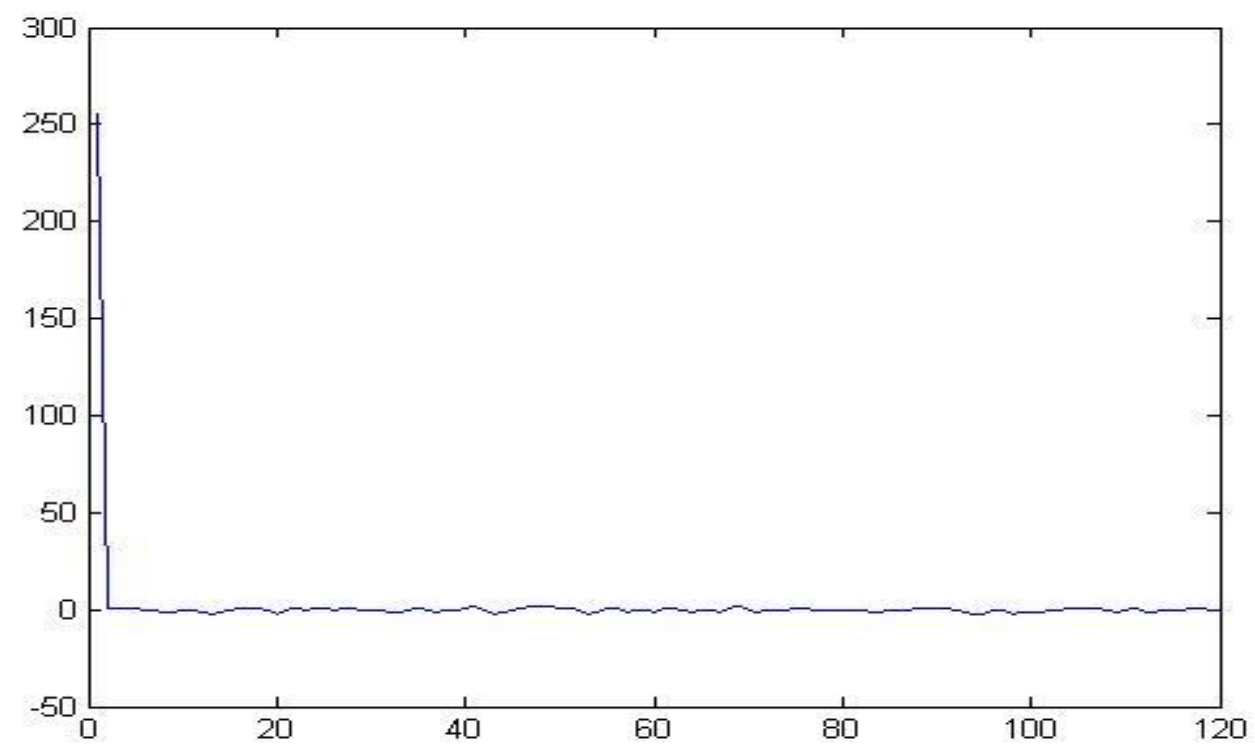

Figure 3.2.5 Auto-correlation of PRBS

\subsubsection{Cross-Correlation of PRBS with Vout with injection of PRBS at Vc node}

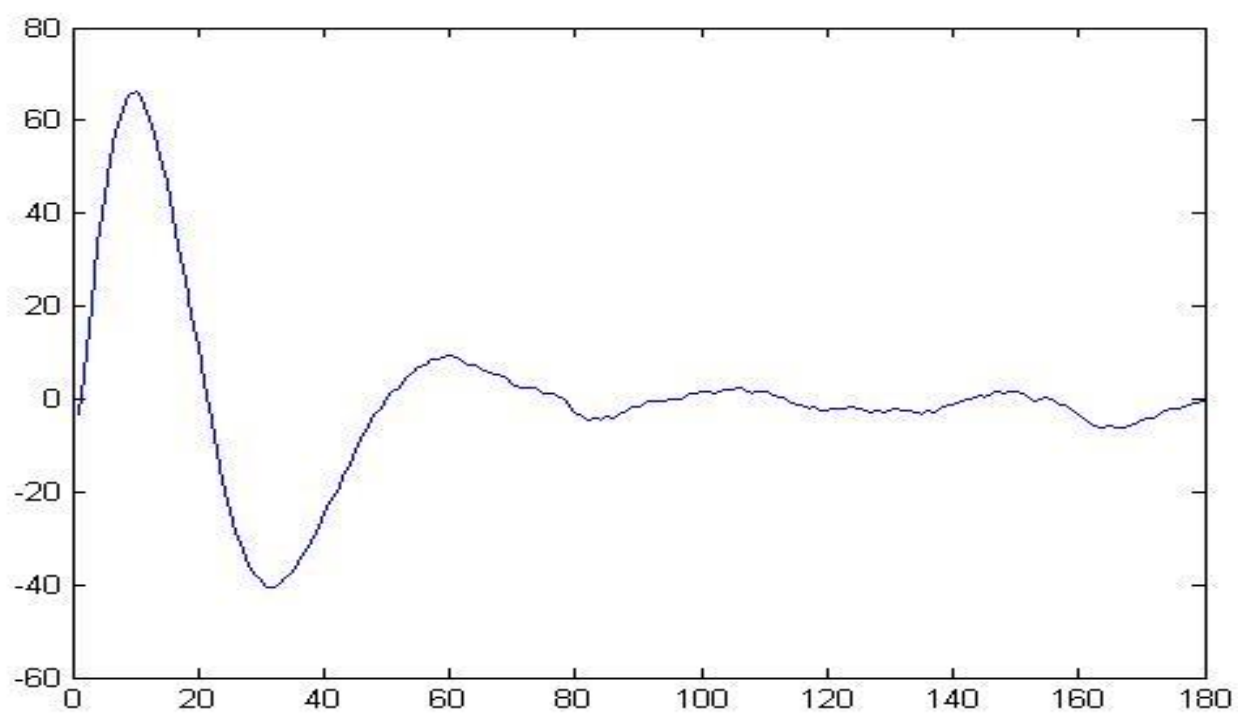

Figure 3.2.6 Cross-Correlation of PRBS with Vout 


\subsubsection{FFT plot of Cross-Correlated result and the Bode plot}

Bode plot of the system from Vc to Vout in closed loop is almost same as the FFT plot of the cross-correlated result. The cross-correlated result is an impulse in time-domain which inherits the system transfer function from Vc to Vout in closed loop.
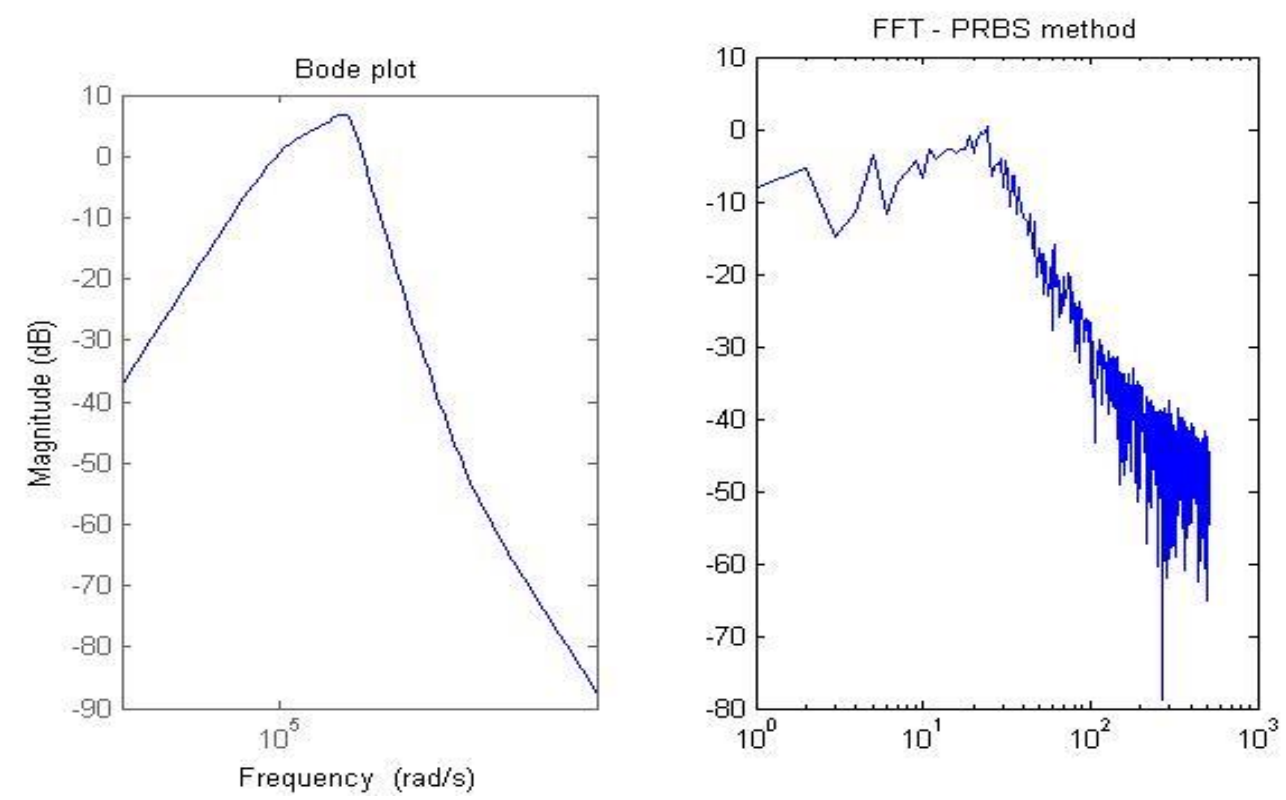

Figure 3.2.7 Bode plot using transfer function and FFT of the time domain impulse using PRBS 


\section{CHAPTER 4}

\section{SELF-TEST METHOD}

\subsection{Proposed Method}

As mentioned in the introduction section, we need a low-overhead method to extract the impulse response. Instead of a full system-ID of the transfer function, we aim to determine the parameters that effect the stability of the converter. These parameters are the natural frequency and the quality factor which are determined by the dominant poles and zeros. Hence, we can limit our observation to within a small region of the entire spectrum. This limitation will simplify the process in two ways: (a) the PRBS frequency can be much lower than the switching rate and (b) multi period PRBS sequence can be used along with time-domain averaging to suppress noise [10].

Figure 4.1.1 shows the proposed dynamic loop characterization methodology. A PRBS generator drives the disturbance at multiple accessible nodes in the DC-DC converter (e.g. reference input node, PWM input node, etc.), the output of the loop filter is correlated with the binary PRBS data using circular correlation technique. This process is repeated multiple times and the results averaged in the time domain to suppress the effects of noise. It should be noted that since the samples of the impulse function are obtained at the peak SNR point after the correlator, this approach has the highest immunity to noise and will provide comparable results to oversampling and post-processing. Additionally the shape of the impulse will be preserved in the presence of process variation but will only result in a DC shift. 


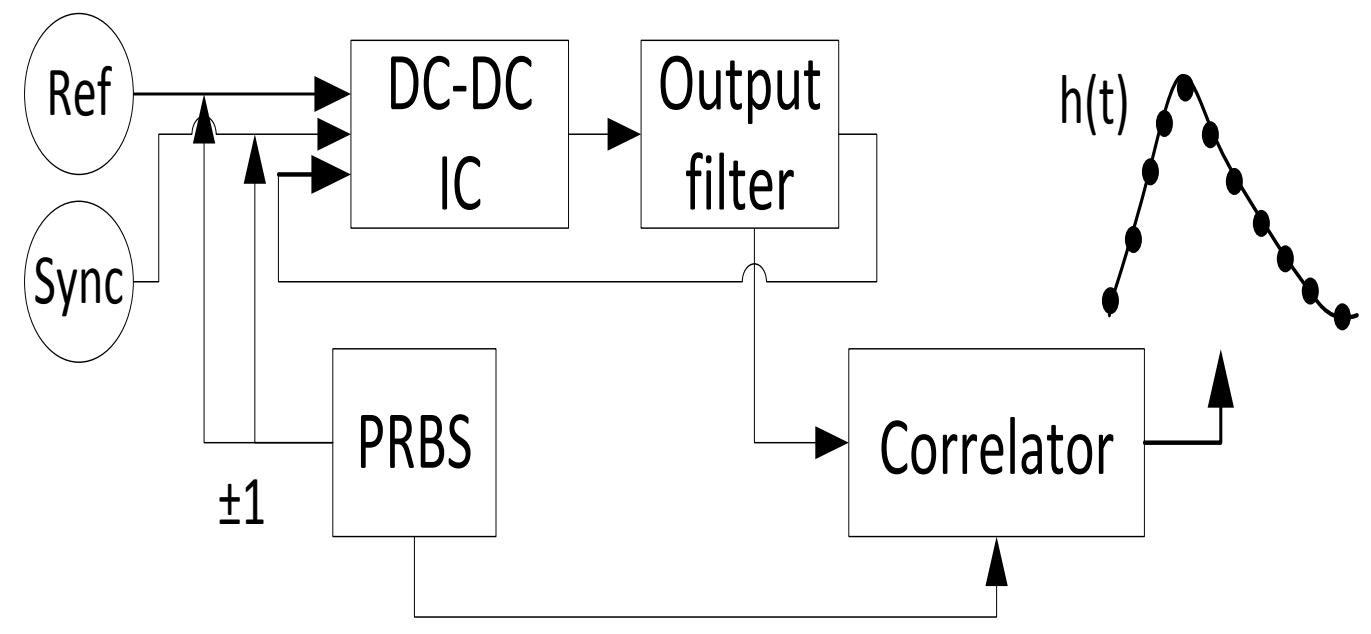

Figure 4.1.1: Proposed dynamic loop characterization methodology

The extracted impulse response function can be processed in two ways. Measure the natural frequency and quality factor directly from the impulse response.

Alternatively, DFT can be used to convert the time domain information into the frequency domain and measure these two parameters. The results from these two techniques are theoretically identical [15].

\subsection{Evaluation Board}

A synchronous single phase PWM DC-DC converter by Texas Instrument, LM27402 evaluation board has been used to experimentally verify the use of the PRBS based identification for the detection of load filter variation. Figure 4.2.1 and 4.2.2 show the evaluation board setup and the converter block diagram with external system identification setup, respectively. Evaluation board consists of a PWM 
controlled DC-DC buck converter IC LM27402, N-type power FETs, L-C output filter, feedback resistive divider ( $R_{F B 1}$ and $\left.R_{F B 2}\right)$, compensation filter $\left(R_{C 1}, R_{C 2}, C_{C 1}, C_{C 2}\right.$ and $\left.\mathrm{C}_{\mathrm{c}}\right)$ and bootstrap capacitor $\mathrm{C}_{\text {boot. }}$. Default component values of the converter on the

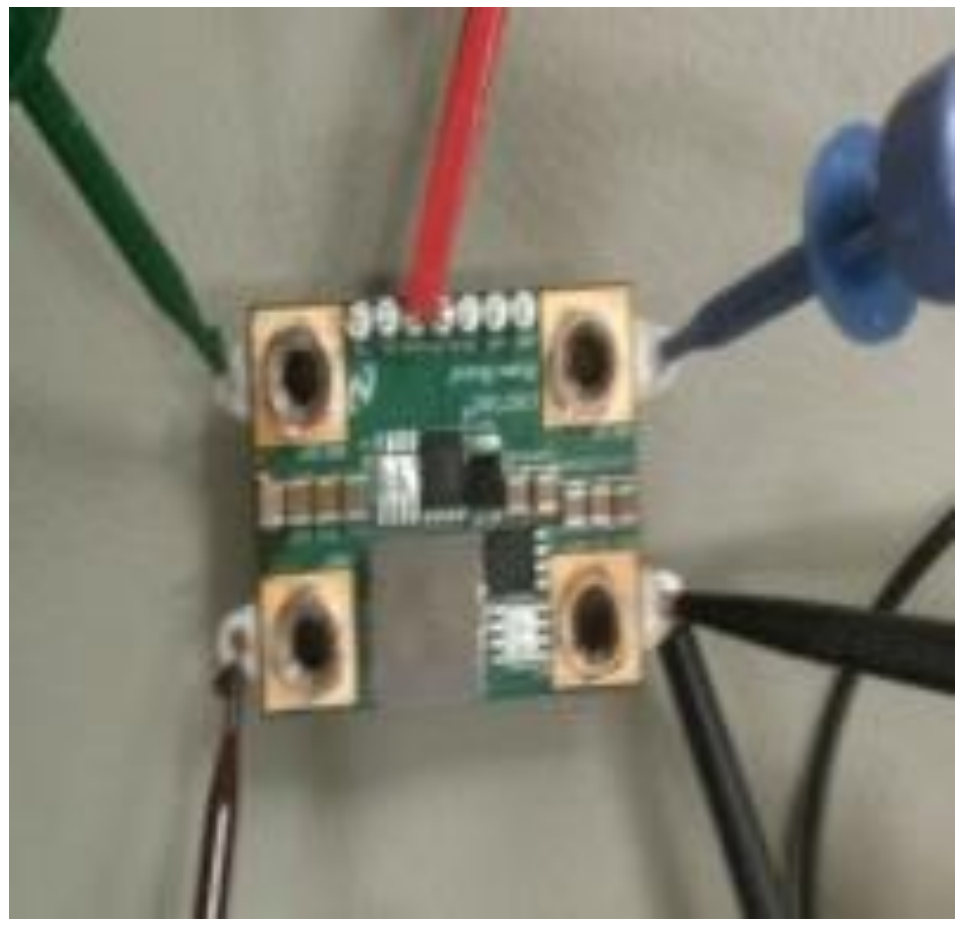

Figure 4.2.1 Evaluation board LM27402 and the experimental set-up

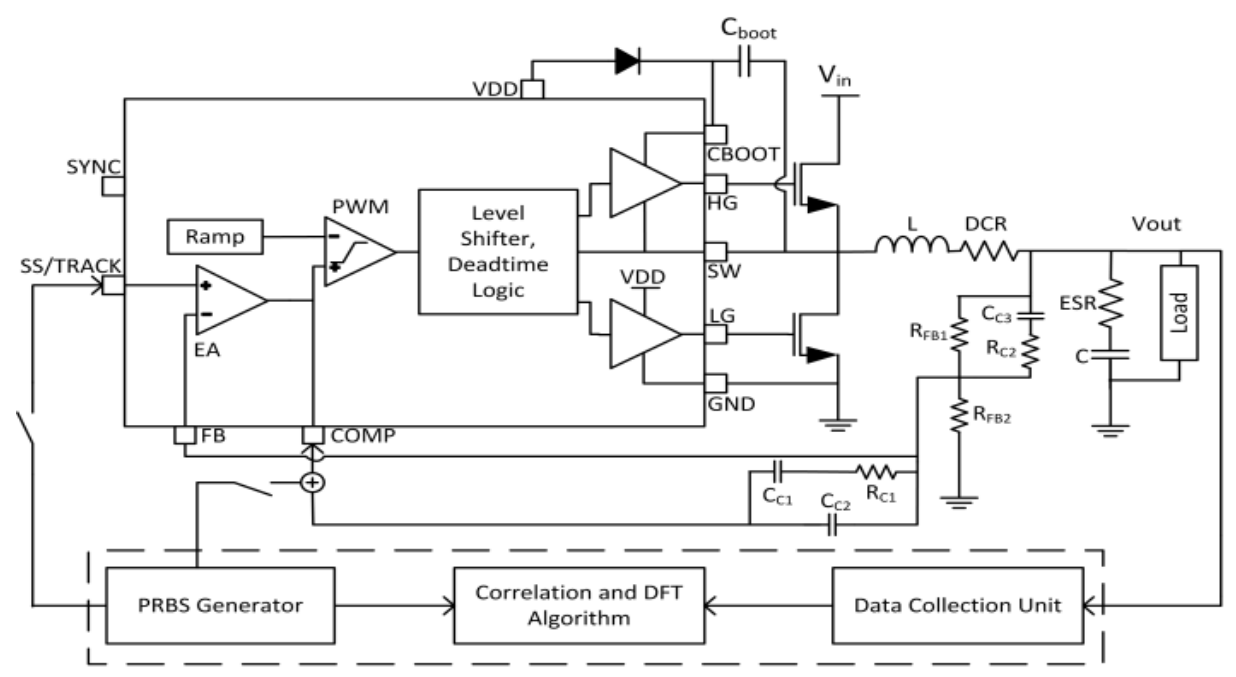

Figure 4.2.2 Simplified block diagram of DC-DC converter on LM27402 Evaluation board with external system identification blocks. 
evaluation board are: output filter inductance $L=0.68 \mu \mathrm{H}$ with $D C R=2.34 \mathrm{~m} \Omega$, output filter capacitor $C=240 \mu \mathrm{F}$ and $E S R=0.75 \mathrm{~m} \Omega, R_{F B 1}=20 \mathrm{k} \Omega, R_{F B 2}=13.3 \mathrm{k} \Omega, R_{C 1}=8.01 \mathrm{k} \Omega$, $\mathrm{R}_{\mathrm{c} 2}=261 \Omega, \mathrm{C}_{\mathrm{c} 1}=3.9 \mathrm{nF}, \mathrm{C}_{\mathrm{c} 2}=150 \mathrm{pF}, \mathrm{C}_{\mathrm{c} 3}=820 \mathrm{pF}, \mathrm{C}_{\mathrm{boot}}=220 \mathrm{nF}$. The transfer function is evaluated at the operating point where the input voltage, $V_{i n}=6.5 \mathrm{~V}$, output voltage, $V_{\text {out }}=1.5 \mathrm{~V}$, load current, $I_{L}=0 A$ and the switching frequency, $f_{s}=300 \mathrm{kHz}$. The PRBS frequency is set at $50 \mathrm{kHz}$, providing an observation bandwidth of $25 \mathrm{kHz}$. From the above nominal values, we can confirm that the dominant poles and zeros of the loop are well within the bandwidth (BW). Although there are higher order poles and zeros, these do not affect the stability of the converter. Moreover, aging will result in degradation of circuit parameters (changing pole/zero locations). Hence, for the purposes of production and in-field testing for the stability, the $25 \mathrm{kHz} B W$ is adequate.

\subsection{Experimental Set-up}

For this experiment, the perturbation signal has been injected through two access nodes: reference input (SS/TRACK pin), to the error amplifier, and the compensated signal input (COMP pin), to the PWM input, of LM27402 controller IC as shown in figure 4.2.2. The response of the converter has been observed at the output node $\left(V_{\text {out }}\right)$. The impulse response obtained from the proposed method is compared with the traditional swept sine-wave method. Output filter variation has been introduced by using four different inductors and three different capacitors that have been removed from the board and replaced. TABLE 4.3.1 shows the parameters of inductors and capacitors. 
TABLE 4.3.1 Load Inductances and Capacitances used in the experiment

\begin{tabular}{|c|c|c|c|c|}
\hline $\mathbf{L}(\mathbf{n H})$ & $\mathbf{\%} \boldsymbol{\Delta L}$ & $\mathbf{D C R}(\mathbf{m} \boldsymbol{\Omega})$ & $\mathbf{C}(\boldsymbol{\mu} \mathbf{F})$ & \% $\boldsymbol{\Delta} \mathbf{C}$ \\
\hline 560 & -18 & 2.3 & 328 & 0 \\
\hline 600 & -12 & 2.3 & 375 & 14 \\
\hline 680 & 0 & 2.34 & 406 & 24 \\
\hline 700 & 3 & 2.5 & - & - \\
\hline
\end{tabular}

PRBS noise has been injected using a function generator. Five periods of maximum length 10 -bit PRBS data with $50 \mathrm{kHz}$ data has been applied. Total PRBS data length is $M=5 \cdot\left(2^{10}-1\right)=5115$. Converter output response at $V_{\text {out }}$, has been captured through a data acquisition block, setup at $50 \mathrm{kHz}$ sampling clock with a 14-bit analog-to-digital converter (ADC) precision controlled by LABVIEW. The PRBS data and captured output data have been transmitted to a PC (personal computer) for the computation of cross-correlation, averaging and DFT based estimation of converter quality factor and natural frequency using MATLAB.

Figure 4.3.1 showing the second order LCR low-pass filter. The ideal part of the cross correlation is simply the discrete-time system impulse response that decays to zero in approximately 50 samples. The non-ideal part doesn't decay over time and contains significant high-frequency content. The precision of the high frequency magnitude in the DFT of cross correlation obtained using PRBS is reduced.

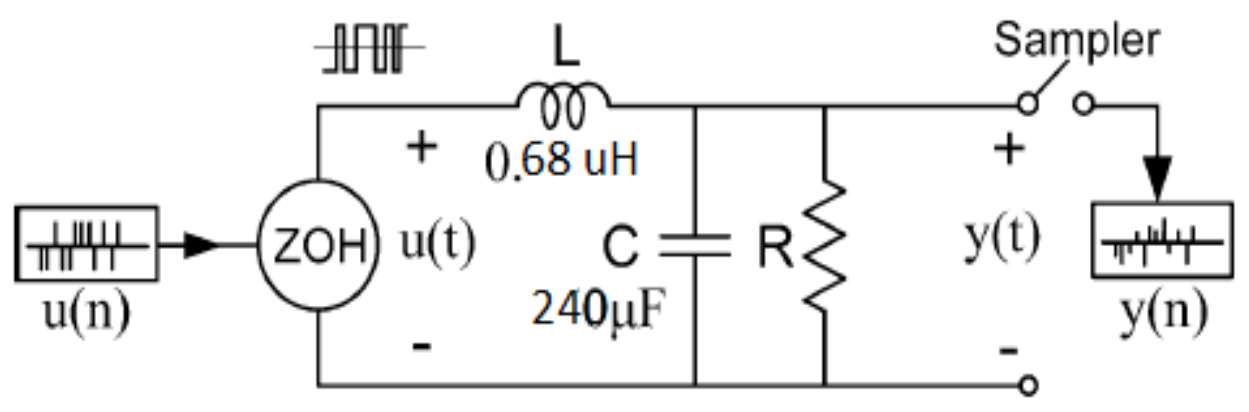

Figure 4.3.1: LCR low-pass filter showing discrete-time to continuous-time interface 
The ultimate goal of this procedure is to estimate the frequency response of a continuoustime system by correlating discrete-time test signal data with the discrete-time sampled system output. A block diagram showing the interfaces between continuous and discrete-time data is shown in figure 4.3.2. The test sequence $u(n)$ is applied to the continuous-time system via a zero-order hold ( $\mathrm{ZOH}$ ) digital-to-analog converter (DAC) and a clock signal, Clock 1. For a power converter, the DAC is a digital pulse width modulator (PWM). The continuous-time output $y(t)$ is sampled using an analog-to-digital converter (ADC) and a clock signal, Clock 2.

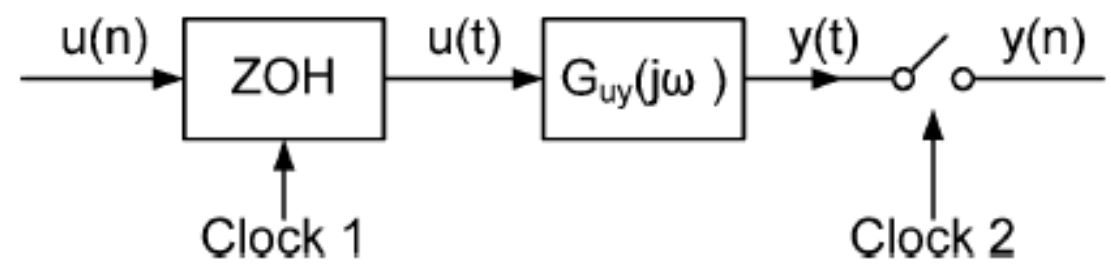

Figure 4.3.2: Block diagram showing the continuous-time plant with the discrete time input and output

\subsection{Transfer Function from Reference Input to Output}

The transfer function from the reference input to the output node includes the output filter, controller poles and zeros, and the ON resistance of the power train transistors. Out of these, the poles and zeros due to the output filter and the ON resistance of the power train fall in-band for the proposed method. These parameters are also subject to the highest levels of process variation and aging. This affect the stability of the system. For the remaining parameters, only catastrophic defects will shift them to within the bandwidth of the proposed measurement, which will be detected. Other parametric variations may affect efficiency, but not stability of the system. This transfer function is effectively a second order transfer function which is slightly under-damped by design. We have used the proposed technique to measure 
the natural frequency $\left(\omega_{0}\right)$ and the quality factor $(Q)$ of this transfer function. For the comparison, we have used the traditional swept sine-wave method where the input node is excited by a single tone sine wave. The gain of the output response is measured by dividing the peak-peak output swing by peak-peak input swing. This process is repeated for multiple frequencies. Input sine-wave has been generated by a function generator and output response has been measured on a high resolution oscilloscope.

Figure 4.4.1 shows the measured transfer function for different inductance values using PRBS method while the comparison between PRBS and sine-wave methods is depicted in figure 4.4.2. The PRBS based response accurately tracks the sine-wave based measured response. Figure 4.4.3 shows the response using swept sine wave with varying inductors.

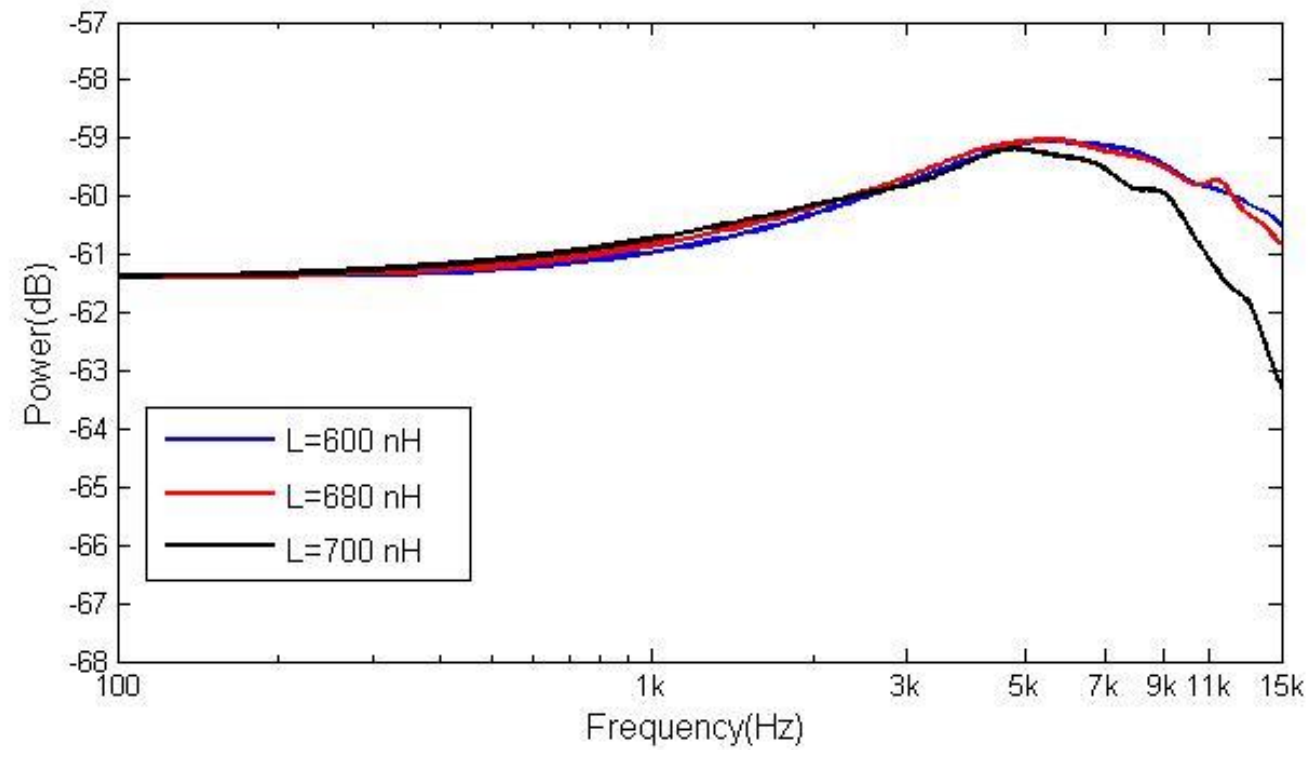

Figure 4.4.1 Frequency response of the system with varying inductance values 


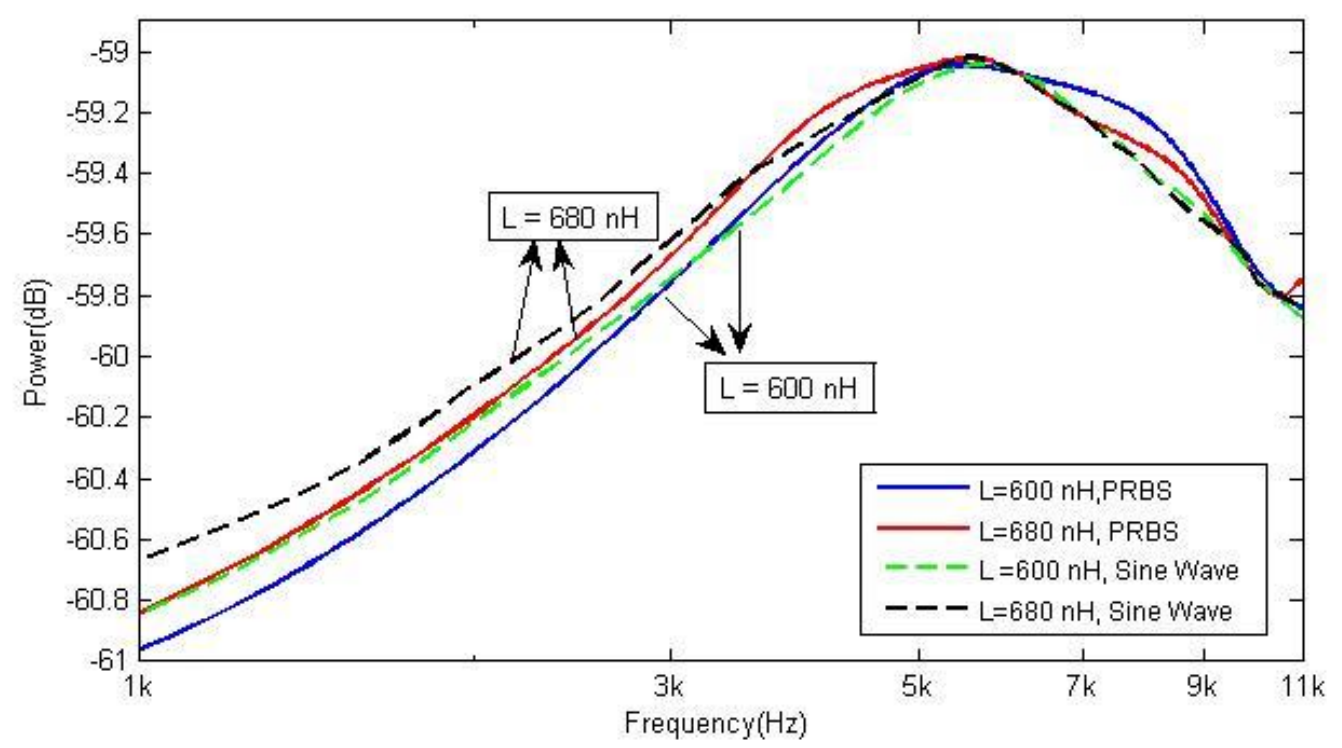

Figure 4.4.2 Overlapping response using PRBS and Swept Sine-Wave

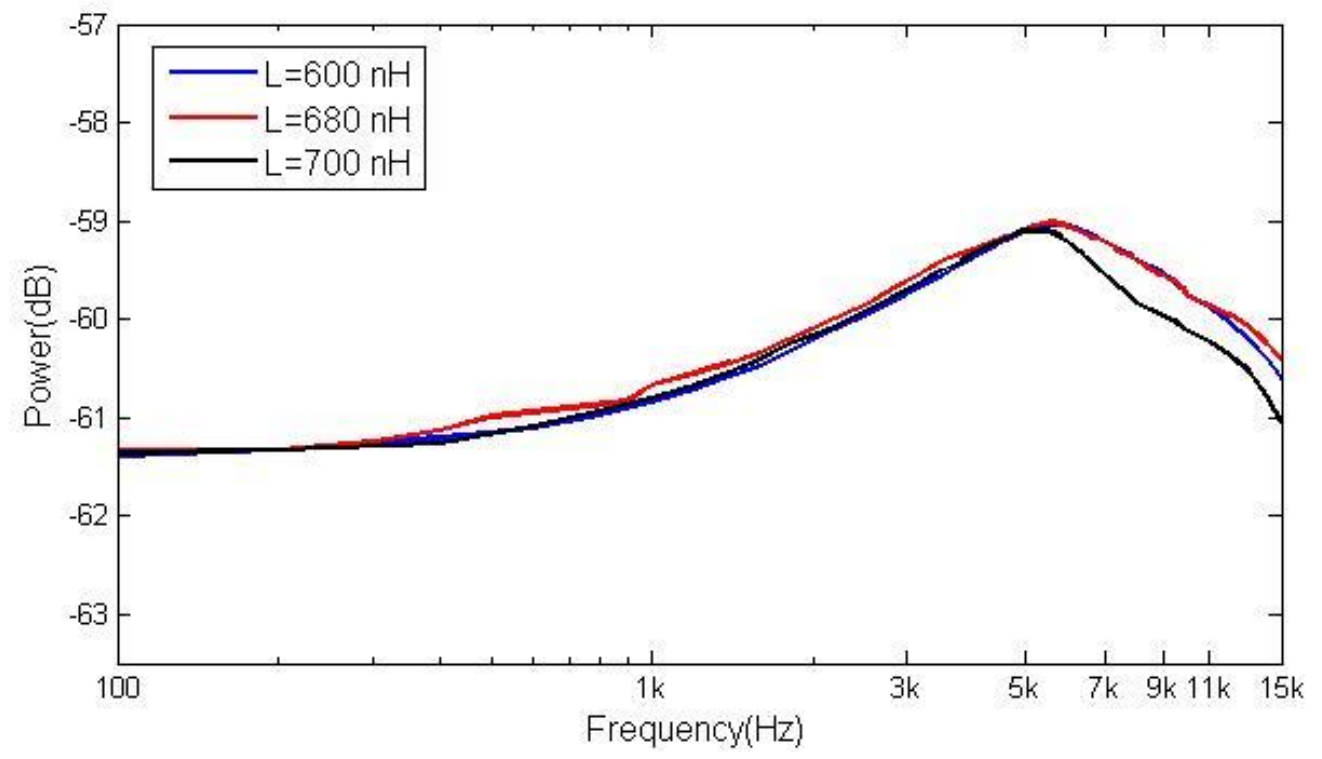

Figure 4.4.3 Swept Sine-Wave response of the system from Vref to Vout 
For both PRBS and sine-wave methods, obtained $\omega_{\circ}$ and $\mathrm{Q}$ values have been tabulated in TABLE 4.4.1 and 4.4.2. TABLE 4.4.2 captures output filter inductance variation results, while TABLE 3 highlights output filter capacitance variation results. The comparison of the PRBS based method with the swept sine-wave method gives less than $\pm 2.5 \% \omega_{\circ}$ error $\left(\varepsilon \omega_{\circ}\right)$ for inductance and capacitance variations. In comparison with the swept sine-wave measurement, the PRBS based Q measurement error $\left(\varepsilon_{Q}\right)$, for the measurement for inductance and capacitance variations, is below $\pm 0.7 \%$.

TABLE 4.4.1 $\omega_{0}$ AND Q, MEASURED FOR DiffeRENT OUtPut Filter INDUCTANCES

\begin{tabular}{|c|c|c|c|c|c|c|}
\hline \multirow{2}{*}{ L (nH) } & \multicolumn{2}{|c|}{ PRBS } & \multicolumn{2}{c|}{ Sine-wave } & \multicolumn{2}{c|}{ Error (\%) } \\
\cline { 2 - 7 } & $\omega_{0}(\mathrm{~Hz})$ & $\mathrm{Q}$ & $\omega_{0}(\mathrm{~Hz})$ & $\mathrm{Q}$ & $\varepsilon_{\omega 0}$ & $\varepsilon_{\mathrm{Q}}$ \\
\hline 600 & 5713 & 1.311 & 5700 & 1.312 & 0.23 & -0.08 \\
\hline 680 & 5469 & 1.315 & 5400 & 1.306 & 1.28 & 0.69 \\
\hline 700 & 4883 & 1.289 & 5000 & 1.294 & -2.34 & -0.39 \\
\hline
\end{tabular}

TABLE 4.4.2 $\omega_{0}$ AND Q, MEASURED for Different OUtPut Filter CAPACITANCES

\begin{tabular}{|c|c|c|c|c|c|c|}
\hline \multirow{2}{*}{ C ( $\boldsymbol{\mu} \mathbf{\mu F})$} & \multicolumn{2}{|c|}{ PRBS } & \multicolumn{2}{c|}{ Sine-wave } & \multicolumn{2}{c|}{ Error (\%) } \\
\cline { 2 - 7 } & $\omega_{0}(\mathrm{~Hz})$ & $\mathrm{Q}$ & $\omega_{0}(\mathrm{~Hz})$ & $\mathrm{Q}$ & $\varepsilon_{\omega 0}$ & $\varepsilon_{\mathrm{Q}}$ \\
\hline 328 & 5469 & 1.315 & 5400 & 1.306 & 1.28 & 0.69 \\
\hline 375 & 5176 & 1.289 & 5300 & 1.294 & -2.34 & -0.39 \\
\hline 406 & 4834 & 1.274 & 4800 & 1.273 & 0.71 & 0.08 \\
\hline
\end{tabular}


It is clear that the proposed method achieves high accuracy in accordance with an industry standard method. As mentioned earlier, the advantage of the proposed method is the ability to spread the test signal energy over the $25 \mathrm{kHz}$ bandwidth and hence not disturb the system operation. To verify that injection of PRBS doesn't add harmonics to the system spectrum, we took the spectral content of output when in normal operation without PRBS. A spectrum analyzer was used to get the spectral content. Spectral content of the output was taken even after injecting PRBS. The two spectrums were overlapped. Figure 4.4.4 shows hardly any change in the output spectrum without and with PRBS noise except the small increase in the noise floor. As the PRBS based method provides negligible disturbance in the output voltage, system identification based on this correlation method is the best suitable technique for in-field assessment of the DC-DC converter.

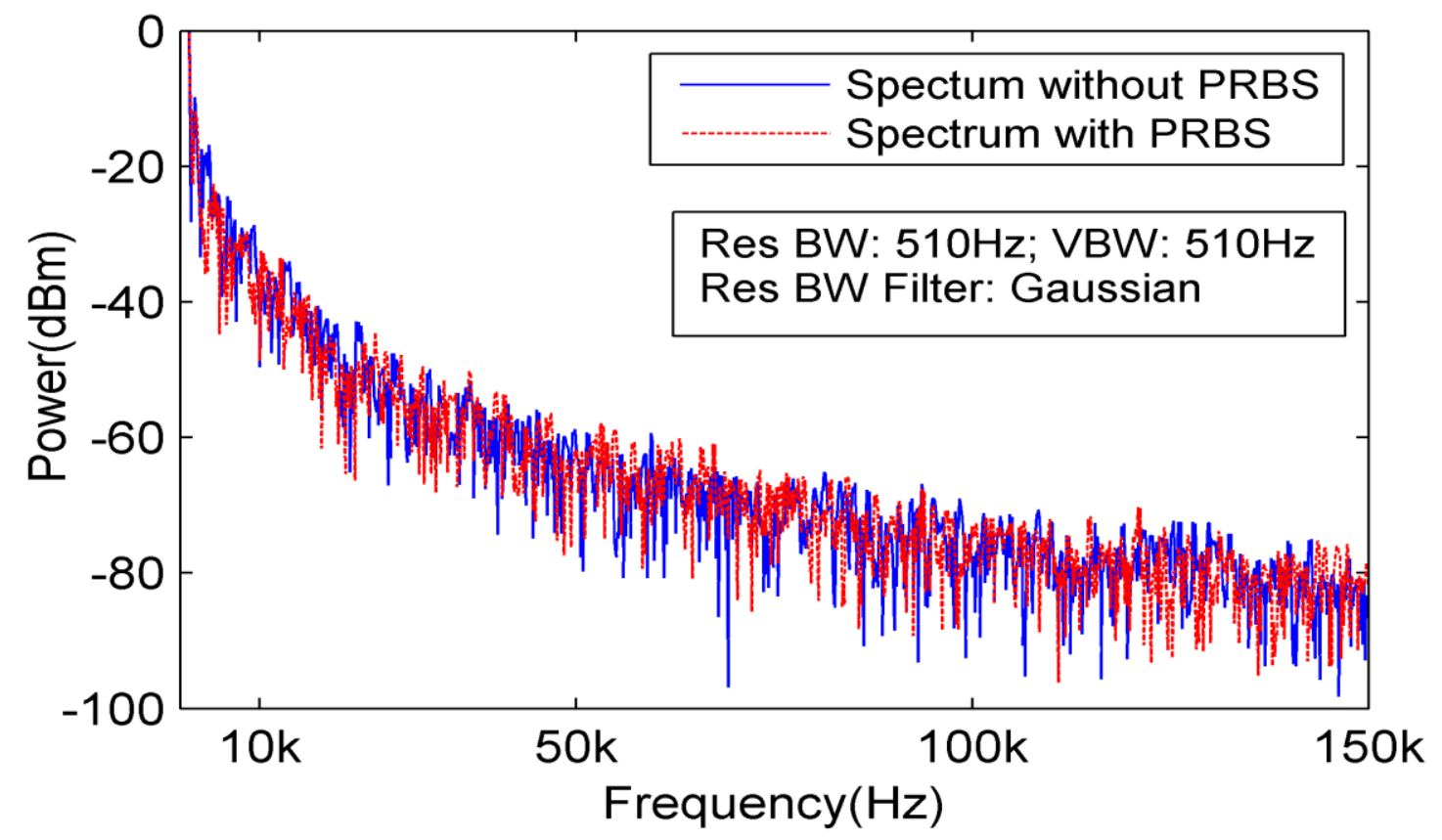

Figure 4.4.4: Spectrum of output voltage $\left(V_{\text {out }}\right)$ with and without PRBS injection at converter reference input (SS/TRACK) for correlation based system identification 


\subsubsection{Response of the System with various capacitance values using PRBS}

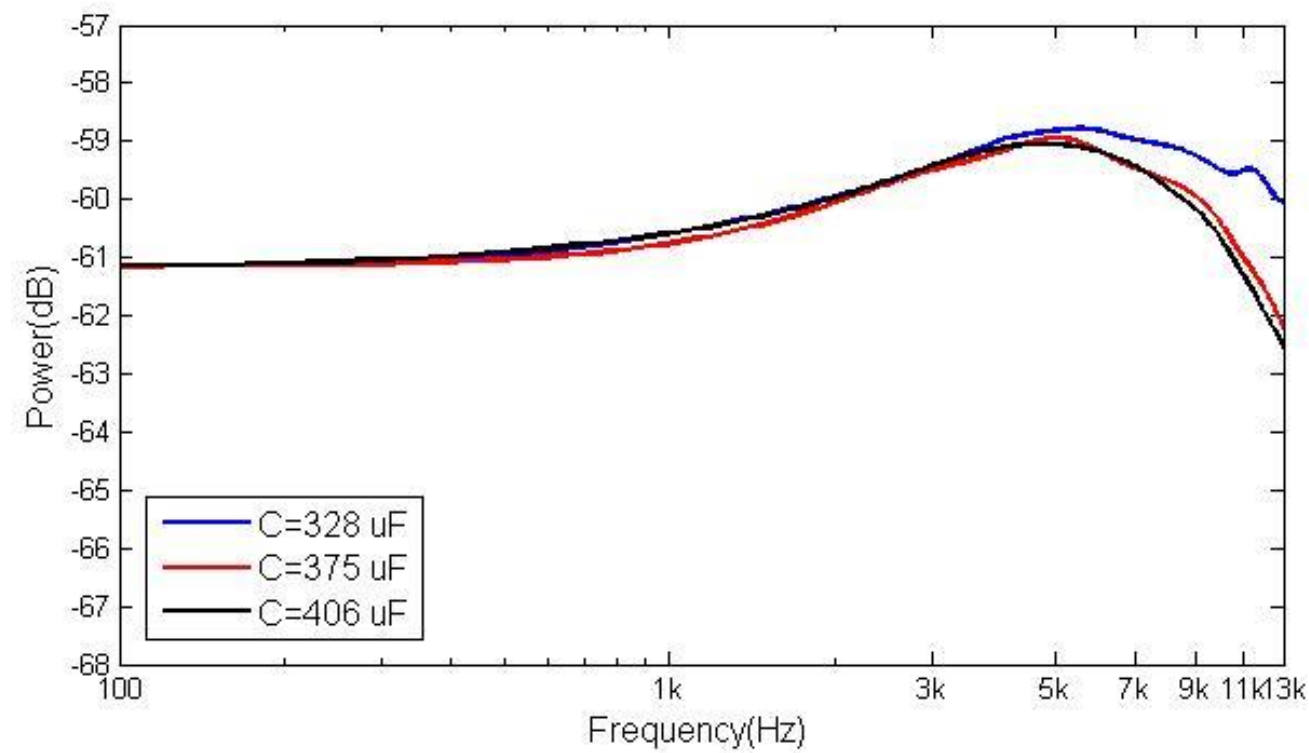

Figure 4.4.5 Frequency Response of the system using PRBS with varying capacitance value

\subsubsection{Response of the system using Swept Sine-Wave with various capacitance values}

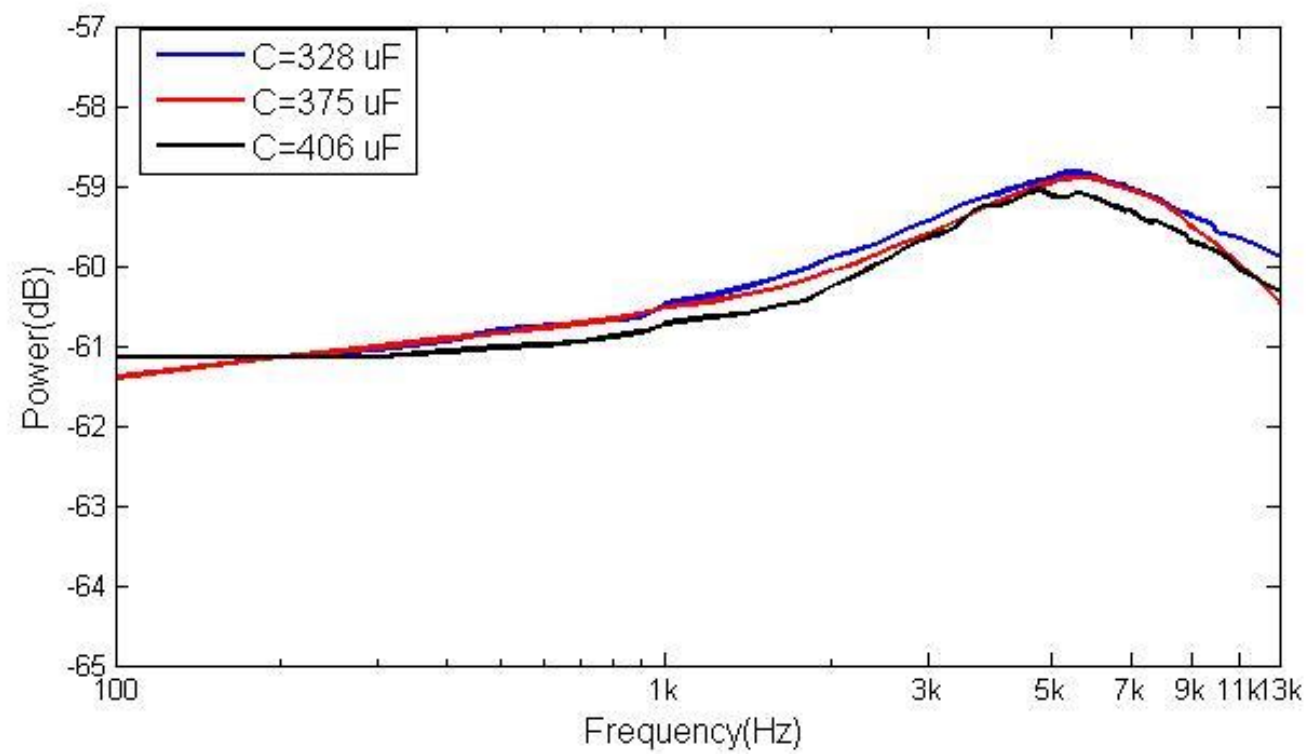

Figure 4.4.6 Response using Swept Sine-Wave with various capacitance 


\subsubsection{Overlapping response using PRBS and Swept Sine-Wave}

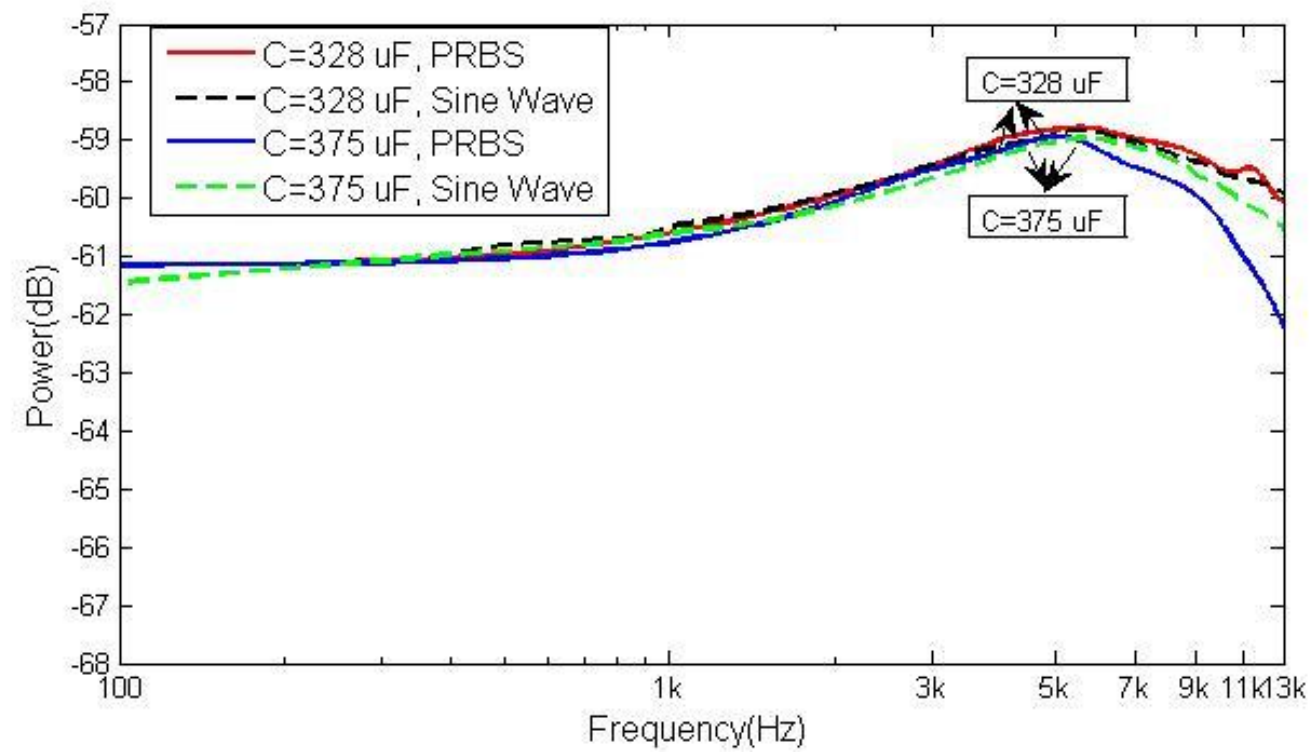

Figure 4.4.7 Overlapping plot showing the PRBS and Swept Sine-Wave response

\subsection{Need for lower sampling frequency}

Extending small-signal models of switching power converters to predict their frequency response at frequencies approaching and beyond half the switching frequency has been an active research topic for more than a decade. Perturbing the duty cycle at a particular frequency $f_{m}$ in a PWM converter running at a switching frequency of $f_{s}$ will:

- Cause significant perturbation responses in the output voltage at (i) the perturbation frequencies, (ii) sum and difference formed from the switching and perturbing frequencies, i.e. $f_{s}-f_{m}$ and $f_{s}+f_{m}$, and (iii) sum and difference frequencies formed from the perturbation frequency and harmonics of the switching frequency, i.e. $2 f_{s}-f_{m}, 2 f_{s}+f_{m}, 3 f_{s}-f_{m}, 3 f_{s}+f_{m}$ etc. 
- Produce the same output perturbation spectrum as a duty ratio perturbation at frequency $\mathrm{kf}_{\mathrm{s}} \pm \mathrm{f}_{\mathrm{m}}$ for integer $\mathrm{k}$;

- Tend to produce the largest perturbation response at the lowest aliased frequency.

\subsection{Transfer Function for PWM Input to Output}

The proposed technique is versatile and low-impact in terms of disturbance. Hence, the same circuit can be used for multiple excitation points to extract more information from the DUT without adding more hardware overhead. We also have the proposed technique to characterize the transfer function from the PWM input to the output. This transfer function has pass-band characteristics and is affected by the output filter, power train transistors, and the compensator poles and zeros. Deviations in this transfer function from its expected response indicate that one or more of the above parameters have drifted. From the earlier characterization, putting the two transfer functions together, it is possible to diagnose the location of the problems in terms of loop components or compensator. Figure 4.6.1 shows the transfer function with varying inductor values while figure 4.6.2 shows with that of capacitance variation. Comparison of transfer function is done between the ones obtained using PRBS method and those from the swept sine-wave method. These results are shown in figure 4.6.3. The results show a very good match between the two methods. Since this is a pass-band behavior, we compare the center frequency $\left(\omega_{c}\right)$ of the two transfer functions. 


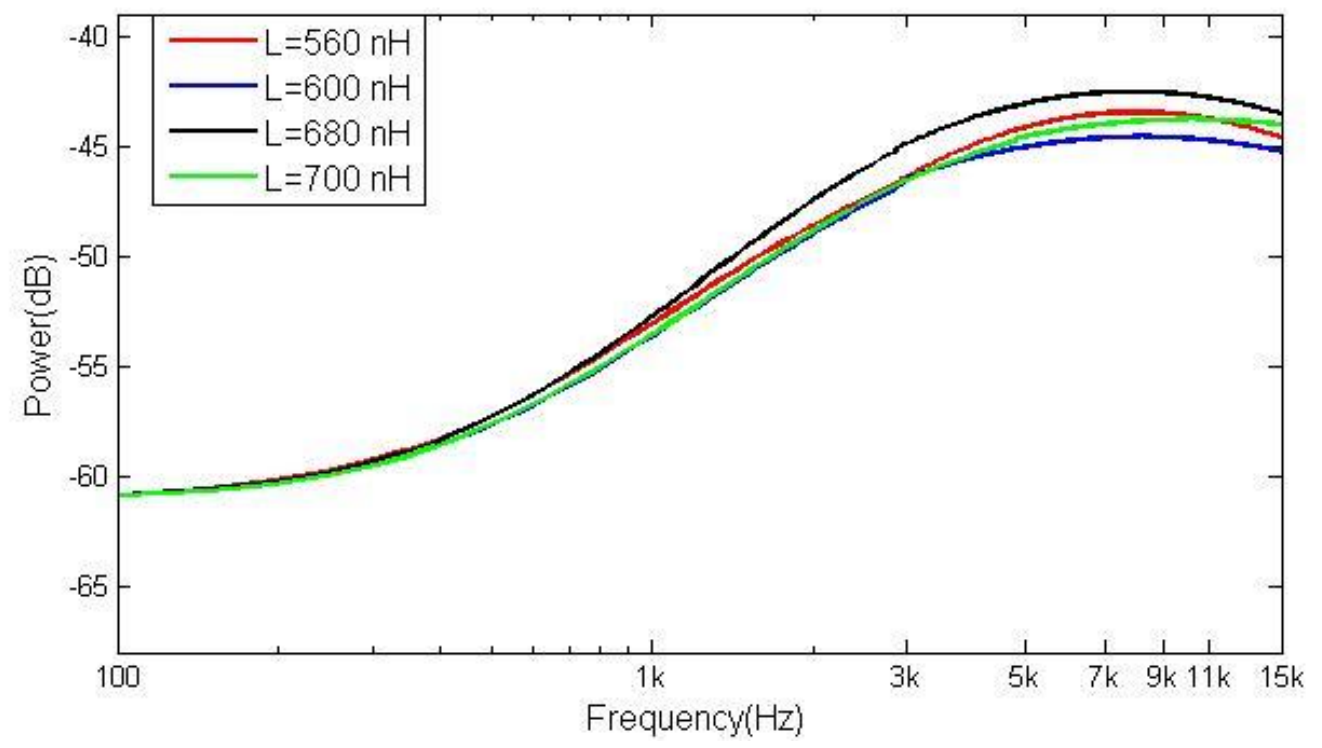

Figure 4.6.1 PWM to Vout transfer function response using PRBS with varying inductance value

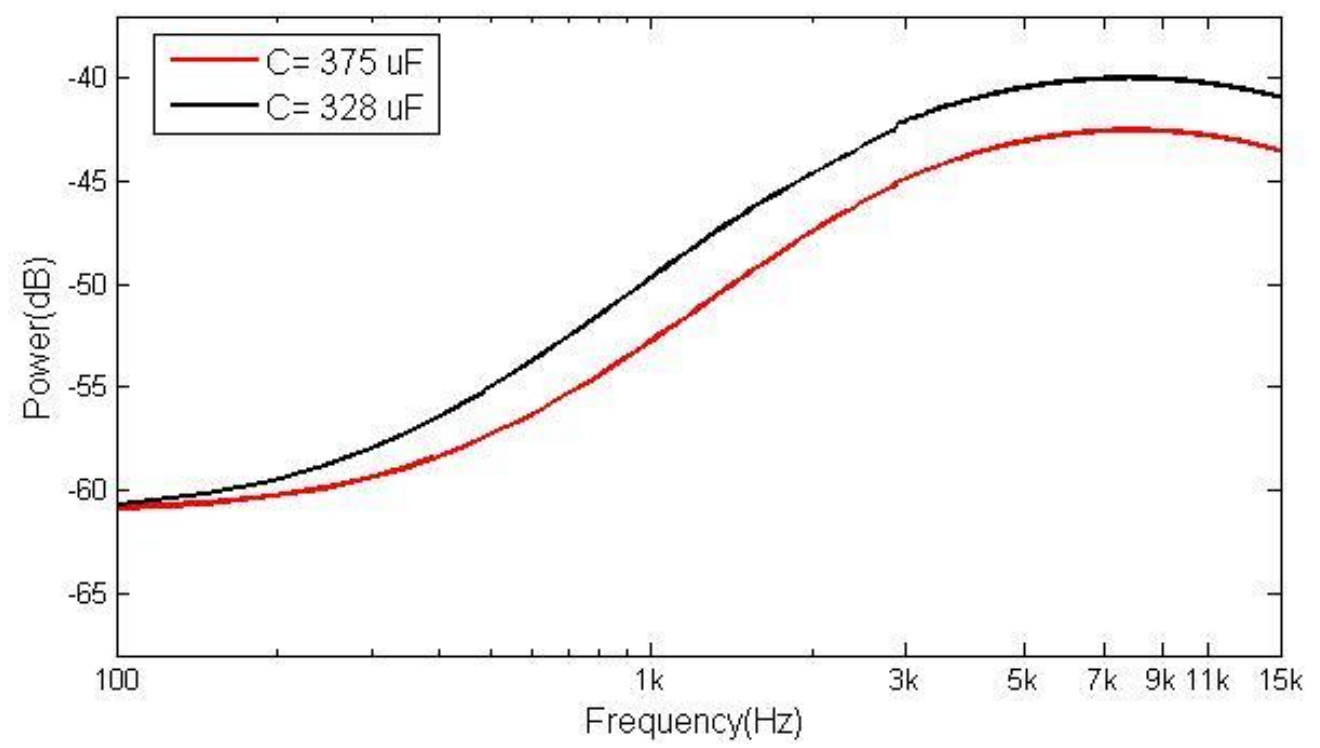

Figure 4.6.2 PWM to Vout transfer function response using PRBS with varying capacitance value 


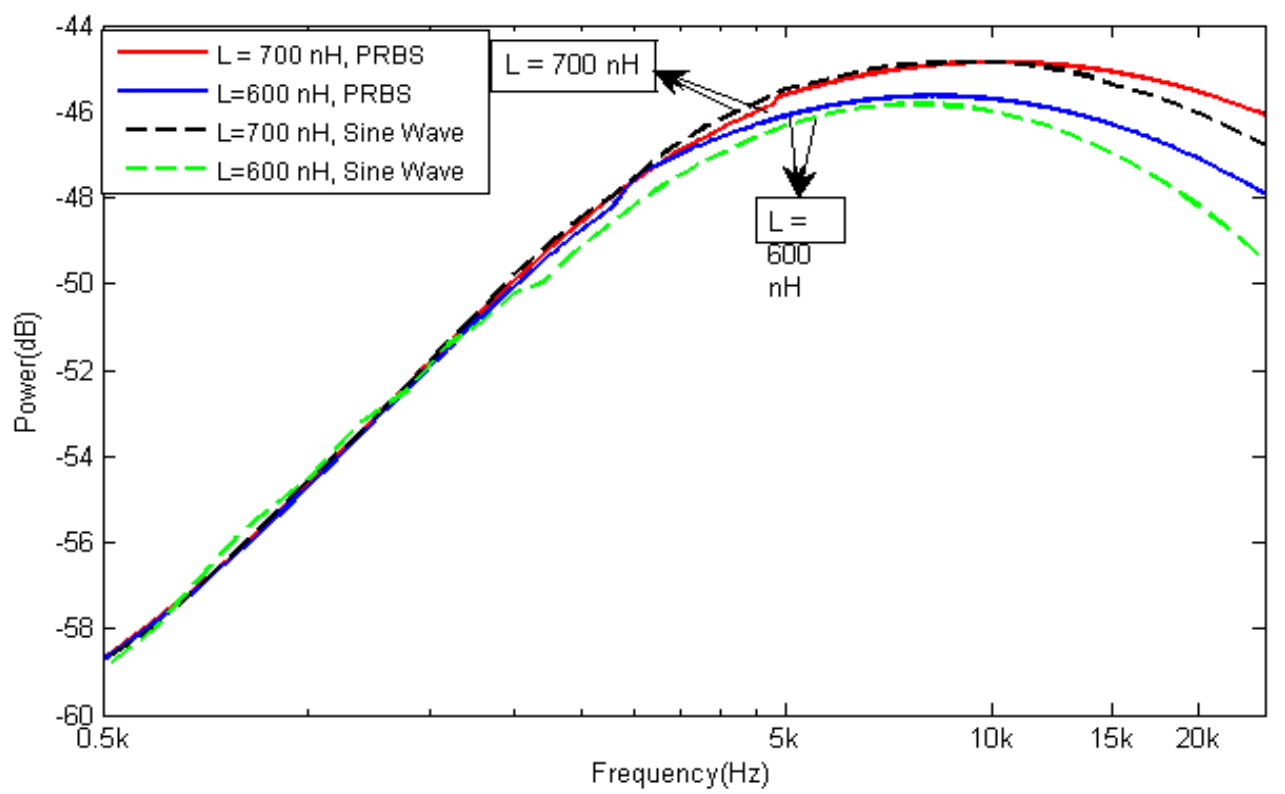

Figure 4.6.3 Overlapping response using PRBS and Swept Sine-Wave

The difference in the absolute gain is irrelevant since it doesn't affect the pole/zero configurations. Figure shows a good matching between the PRBS and sinewave based measurements. Corresponding $\omega_{c}$ values obtained by the PRBS and swept sine-wave measurements have been summarized in TABLE 4.6.1 for different output filter inductances and in TABLE 4.6.2 for different output filter capacitances. Inductances and capacitances are of the same values as noted in TABLE 4.3.1. With respect to swept sine-wave, in PRBS method the measurement errors in $\omega_{c}\left(\varepsilon \omega_{c}\right)$ over $L$ and $C$ variations are lower than $\pm 0.8 \%$.

TABle 4.6.1 $\omega_{c}$ MeAsured for Different OutPut Filter Inductances

\begin{tabular}{|c|c|c|c|}
\hline \multirow{2}{*}{$\mathbf{L}(\mathbf{n H})$} & PRBS & Sine-wave & Error (\%) \\
\cline { 2 - 4 } & $\omega_{\mathrm{c}}(\mathrm{Hz})$ & $\omega_{\mathrm{c}}(\mathrm{Hz})$ & $\boldsymbol{\varepsilon}_{\omega c}$ \\
\hline 560 & 8008 & 8000 & 0.10 \\
\hline 600 & 8252 & 8200 & 0.63 \\
\hline 680 & 8545 & 8600 & -0.64 \\
\hline 700 & 9570 & 9500 & 0.74 \\
\hline
\end{tabular}


TABLE 4.6.2 $\omega_{c}$ MEASURED for DiffERENT OUtPUt Filter CAPACITANCES

\begin{tabular}{|c|c|c|c|}
\hline \multirow{2}{*}{$\mathbf{C}(\boldsymbol{\mu} \mathbf{F})$} & PRBS & Sine-wave & Error (\%) \\
\cline { 2 - 4 } & $\omega_{c}(\mathrm{~Hz})$ & $\omega_{c}(\mathrm{~Hz})$ & $\boldsymbol{\varepsilon}_{\omega c}$ \\
\hline 328 & 8545 & 8600 & -0.64 \\
\hline 375 & 7959 & 7900 & 0.75 \\
\hline
\end{tabular}




\section{CHAPTER 5}

\section{THESIS SUMMARY AND FUTURE WORK}

\subsection{Summary}

High frequency switching converters have been gaining prominence in the DC-DC converter market due to their high efficiency. Unfortunately, they are also subject to higher process variations jeopardizing stable operation of the power supply. This thesis talks about a technique to track changes in the dynamic loop characteristics of the DC-DC converters without disturbing the normal mode of operation using a white noise based excitation and correlation. White noise excitation is generated via pseudo random disturbance at reference and control input of the converter with the test signal energy being spread over a wide bandwidth, below the converter noise and ripple floor. The variation in loop characteristics is determined using a PRBS-based small perturbation white noise. The results are compared with those obtained using frequency swept sine-wave method. The technique is independent of the converter type and can be used without impacting the normal operation of the converter, during the closed-loop operation.

The proposed technique is demonstrated on a TI LM27402 switch mode buck converter. The results obtained by PRBS method with the injection point at error amplifier input are within $\pm 2.5 \%$ and $\pm 0.7 \%$ for $\omega_{\circ}$ and $Q$, respectively, over variation in inductance and capacitance. The error is less than $\pm 0.8 \%$ for $\omega_{c}$ over inductance and capacitance variation when the injection point is at the PWM input. Single bit PRBS injection enables digital friendly implementation on silicon. 


\subsection{Test time and Silicon Overhead}

The overall time for transfer function extraction is determined by the PRBS frequency, the number of patterns in the PRBS signal, and the number of points evaluated on the time domain transfer function. While these numbers will depend on the individual DUT, the overall test time for the experimental circuit is about $100 \mathrm{~ms}$. It should be noted that the proposed technique works in the background, without disturbing the converter operation. Hence, the test time is not of high importance.

The technique requires IC implementation of (a) PRBS generator, (b) analog correlator. Hence, the proposed method has numerous advantages compared to techniques proposed in the literature. The proposed technique requires only binary excitation and binary correlation. This greatly reduces the hardware overhead of the entire measurement system and leads to easier, switchedcapacitor based implementation. 


\section{REFERENCES}

[1]. N.Kurd et al., "Haswell: A Family of IA 22nm processors," in IEEE Int. Solid State Circuits Conf. Dig. Tech. Papers, 2014, pp. 112-113

[2]. T.Liu et al., "A built-in self-test technique for load inductance and lossless Current sensing of DC-DC converters," in Proc. IEEE VLSI Test Symp.,2014, pp. 1-6.

[3]. L. Ljung, System Identification: Theory for the User, $2^{\text {nd }}$ ed. Englewood Cliffs, NJ: Prentice-Hall, 1999.

[4]. G. F. Franklin, J. D. Powell, and M.L. Workman, Digital Control of Dynamic Systems, $3^{\text {rd }}$ ed. New York: Addison-Wesley, 1997.

[5]. D. Maksimovic, "Computer-aided small-signal analysis based on impulse response of DC/DC switching power converters," IEEE Trans. Power Electron., vol. 15, no.6, pp. 1183-1191, Nov, 2000.

[6]. B. Johansson and M. Lenells, "Possibilities of obtaining small-signal models of DC-to-DC power converters by mean of system identification," in Proc. Telecommunications Energy Conf., 2000, pp. 65-75.

[7]. P. Huynh and B. H. Cho, "Empirical small-signal modeling of switching converters using PSpice", in Proc. IEEE PESC"95, 1995, pp. 801-808.

[8]. A. Costabeber, P. Mattavelli, et al., "Digital Autotuning of DC-DC Converters Based on a Model Reference Impulse Response", IEEE Transactions on Power Electronics, vol. 26, no. 10, pp. 2915-2924.

[9]. X. Wang, K. Blanchand, et al., "Alternative "safe" test of hysteretic power converters, "IEEE VLSI Test Symposium, pp 1-6, Apr, 2014.

[10]. B. Miao, R. Zane, et al., "System Identification of Power Converters With Digital Control Through Cross-Correlation Methods", IEEE Transactions on Power Electronics, vol. 20, no. 5, pp. 1093-1099.

[11]. A. Barkley and E. Santi, "Improved Online Identification of a DC-DC Converter and its Control Loop Gain Using Cross-Correlation Methods", IEEE Trans. On Power Electronics, vol. 24, no. 8, pp. 2021-2031, Aug2009.

[12]. T. Roinila, T. Helin, et al., "Circular correlation based identification of Switching power converter with uncertainty analysis using fuzzy density Approach", Simulation Modelling Practice and Theory, vol. 17, pp. 1043-1058, 2009.

[13]. R. W. Erickson and D. Maksimovic, Fundamentals of Power Electronics, $2^{\text {nd }}$ ed. Boston, MA: Kluwer, 2001. 
[14]. K. R. Godfrey, "Introduction to binary signals used in system identification", in Proc. Int. Conf. Control, vol. 1, 1991, pp. 161-166.

[15]. B. H. Karnopp and F. E. Fisher, "On the vibrations of overdamped systems", Journal of the Franklin Institute, vol. 327, no. 4, pp. 601-609, 1990. 